\title{
On the baroclinic instability of axisymmetric rotating gravity currents with bottom slope
}

\author{
By PAUL F. CHOBOTER AND GORDON E. SWATERS \\ Applied Mathematics Institute, Department of Mathematical Sciences and Institute for \\ Geophysical Research, University of Alberta, Edmonton, T6G 2G1, Canada
}

(Received 25 January 1999 and in revised form 23 November 1999)

The baroclinic stability characteristics of axisymmetric gravity currents in a rotating system with a sloping bottom are determined. Laboratory studies have shown that a relatively dense fluid released under an ambient fluid in a rotating system will quickly respond to Coriolis effects and settle to a state of geostrophic balance. Here we employ a subinertial two-layer model derived from the shallow-water equations to study the stability characteristics of such a current after the stage at which geostrophy is attained. In the model, the dynamics of the lower layer are geostrophic to leading order, but not quasi-geostrophic, since the height deflections of that layer are not small with respect to its scale height. The upper-layer dynamics are quasi-geostrophic, with the Eulerian velocity field principally driven by baroclinic stretching and a background topographic vorticity gradient.

Necessary conditions for instability, a semicircle-like theorem for unstable modes, bounds on the growth rate and phase velocity, and a sufficient condition for the existence of a high-wavenumber cutoff are presented. The linear stability equations are solved exactly for the case where the gravity current initially corresponds to an annulus flow with parabolic height profile with two incroppings, i.e. a coupled front. The dispersion relation for such a current is solved numerically, and the characteristics of the unstable modes are described. A distinguishing feature of the spatial structure of the perturbations is that the perturbations to the downslope incropping are preferentially amplified compared to the upslope incropping. Predictions of the model are compared with recent laboratory data, and good agreement is seen in the parameter regime for which the model is valid. Direct numerical simulations of the full model are employed to investigate the nonlinear regime. In the initial stage, the numerical simulations agree closely with the linear stability characteristics. As the instability develops into the finite-amplitude regime, the perturbations to the downslope incropping continue to preferentially amplify and eventually evolve into downslope propagating plumes. These finally reach the deepest part of the topography, at which point no more potential energy can be released.

\section{Introduction}

In the world's oceans, mesoscale gravity currents are geostrophically balanced flows on a sloping bottom which are driven by the density difference between the relatively dense current itself and the surrounding water. These currents may occur when dense water is formed or otherwise released into a shallow sea with a sloping bottom, such as a continental shelf region, and settles to the bottom. There, the combined influences of the Coriolis and buoyancy stresses may force the current 
to be transversely constrained and flow along the shelf such that, in the Northern Hemisphere, shallow water is to its right. Examples of such flows include the initial migration of Antarctic bottom water (Whitehead \& Worthington 1982), deep water formation in the Adriatic Sea (Zoccolotti \& Salusti 1987) and deep water exchange in the Strait of Georgia (LeBlond et al. 1991). (For a general review of convective processes in the oceans, see Maxworthy 1997, and for a review of the effects of rotation on surface or bottom-dwelling gravity currents, with or without bottom slope, see Griffiths 1986.)

Griffiths, Killworth \& Stern (1982) investigated the effects of rotation on the stability of a gravity current on a sloping bottom. Their analytical investigation included a low-wavenumber expansion of a single-layer reduced-gravity model. This analysis was compared with experiments they performed on the stability of a surface-dwelling current within a flat-bottomed tank. The results of their analysis and their experimental observations differed on a few key points, e.g. the instability occurred over finite wavenumbers, and a dipole-like mode was observed but not predicted theoretically. The discrepancies between the observations and the theory were attributed to the possible presence of an unstable baroclinic mode which could not be described by the reduced-gravity model of Griffiths et al. (1982).

Partly in order to address these issues, analytical models have been constructed to investigate such flows, such as in Swaters (1991; see also Swaters \& Flierl 1991 and Whitehead et al. 1990). They derived a two-layer model to describe the baroclinic evolution of rotating density-driven currents on a sloping bottom. The model corresponds to a sub-inertial approximation to the two-layer shallow-water equations, where the upper layer is assumed to be quasi-geostrophic and its dynamics are driven by baroclinic vortex tube stretching and the background topographic vorticity gradient. The lower-layer dynamics are geostrophic to leading order, but allow for finite-amplitude thickness variations, since the deflections of the lower-layer height are of the same order of magnitude as its scale height. Barotropic instabilities are filtered out of the model, so as to focus on the baroclinic processes, i.e. the instabilities are driven by the release of the available gravitational potential energy associated with the downslope position of the centre of mass of the gravity current.

Swaters (1991) examined the linear instability characteristics of the model in the case where the lower layer takes the shape of a coupled front. It was found that solutions take the form of along-front travelling waves, with preferential amplification of the perturbations on the down-slope side of the current. Swaters (1991) also noted that the model successfully predicts the dipole mode observed by Griffiths et al. (1982). From a modal point of view, the instability may be thought of as the coalescence of two topographic vorticity waves which have been excited in the upper layer.

The cross-slope asymmetry of the unstable mode is characteristic of baroclinic instability, and is a manifestation of the release of the available potential energy. A number of numerical simulations (e.g. Gawarkiewicz \& Chapman 1995; Chapman \& Gawarkiewicz 1995; Jiang \& Garwood 1995, 1996; Swaters 1998a) have also shown that the spatial structure of the baroclinic instabilities associated with density-driven flows on a sloping bottom are strongly asymmetrical in the cross-slope direction. This is in contrast to instabilities of surface-driven currents (on an $f$-plane), where there is no external force acting to break the underlying cross-current symmetry. This helps to explain, in part, the discrepancy between the theoretical predictions and the laboratory experiments described by Griffiths et al. (1982).

The instabilities observed by Swaters (1991) develop into downslope propagating plumes, and eventually into alongslope propagating cold-core eddies. Swaters (1998a) 
described numerical simulations of the model for coupled fronts and eddies, and documented the evolution of the downslope plumes into alongslope propagating eddies. The propagation characteristics of these eddies have been studied by Swaters \& Flierl (1991) and Swaters (1998b). The nonlinear stability of steady solutions to the model have been examined by Swaters (1993) and Karsten \& Swaters (1996) by exploiting the underlying non-canonical Hamiltonian structure of the model. This model has also been employed to study the stability characteristics of deep water replacement in the Strait of Georgia (Karsten, Swaters \& Thomson 1995). Recently, the model has been extended to allow for a continuously-stratified upper layer (Poulin $\&$ Swaters $1999 a, b, c)$.

In the laboratory, mesoscale gravity currents have been reproduced in rotating tanks with sloping bottoms. For example, Smith (1977) generated bottom currents via a localized source of dense fluid on an axisymmetric slope. In the parameter regime of low viscosity, the current was seen to immediately break up into a series of eddies. This was also observed by Condie (1995) for low viscosity and intermediate rotation rates, as well as Etling \& Chabert d'Hieres (1997) for high rotation rates, low slope, low density excess and a weak source rate. Similar laboratory observations of eddy production by a constant source of dense fluid have been made by Nagata et al. (1993) and Zatsepin, Didkovski \& Semenov (1998). (For laboratory studies of the propagation characteristics of the eddies themselves, see Mory, Stern \& Griffiths 1987 and Whitehead et al. 1990.) Fluid behaviour in this parameter regime seems to agree with the numerical simulations of Jiang \& Garwood (1996) as well as the instability predictions of Swaters (1991, 1998a).

Lane-Serff \& Baines (1998) performed experiments where a dense fluid is allowed to flow over a weir and down a slope. They observe the flow to become geostrophically constrained and immediately break up into a series of eddies, which propagate along the slope. They demonstrate that vortex stretching in the upper layer plays an important role in the formation of eddies (which agrees well with the observations and numerical simulations of Denmark Strait overflow variability by Spall \& Price 1998), and demonstrate that Ekman drainage is an important cause of the vortex stretching.

Lane-Serff \& Baines (1998) argue that baroclinic instability is not significant to eddy formation in this context by showing that the interaction parameter (denoted $\mu$, see below) of Swaters (1991) is not correlated with the observed time interval between eddies. We will demonstrate that, not only does the Swaters (1991) model predict that those two variables should be uncorrelated, but it predicts that the correlation is instead between the interaction parameter and a scaled time interval between eddies. Furthermore, we show that the data of Lane-Serff \& Baines (1998) quantitatively confirms the predicted dependence for the parameter regime in which the Swaters (1991) model is designed to apply.

Ungarish \& Huppert (1998) recently studied the effects of rotation in the axisymmetric initial value problem (where a dense fluid is released from rest at the centre of a cylindrical rotating tank) and found that even a slight rotation has the pronounced effect of halting the radial flow and developing a geostrophic balance within half a rotation of the tank. Although their experiments were performed without a sloping bottom, centrifugal forces create an effective axisymmetric parabolic bottom topography. (This effect provided the only bottom topography in the experiments of Condie 1995.)

Ungarish \& Huppert (1998) point out that the steady-state lens shape that the fluid attains should be considered to be only quasi-steady, i.e. it is ultimately an unstable 
configuration. Given the environmental importance of these near-geostrophic flows and the practicality of conducting experiments on rotating gravity currents in rotating cylindrical tanks, it is of interest to determine the instability characteristics of these flows in this geometrical configuration. The principal purpose of this paper is to describe the baroclinic instability of this current configuration when a sloping bottom is present.

In this paper we modify the Swaters (1991) model to investigate the instability of an initially axisymmetric bottom-dwelling annulus current along a sloping bottom in a rotating cylindrical geometry. The analogy between stability characteristics in rectangular and cylindrical geometries was also addressed by Waugh \& Dritschel (1991), who studied the shear instability of strips of uniform potential vorticity in various two-dimensional models. Their results include the fact that the dispersion relation for a thin circular strip reduces to that for a linear strip in the limit as the width of the strip goes to zero. We shall demonstrate that a similar result holds for the two-layer (i.e. baroclinic) model we employ here.

Our findings include that the baroclinic instability of an axisymmetric current is most influenced by a parameter, denoted $\mu$, which represents the ratio of the scale height of the bottom current to the scale height of the bottom topography. Dynamically, this parameter measures the ratio of the destabilizing effect of vortex stretching to the stabilizing effect of the sloping topography (Swaters 1991). In addition to describing numerical simulations of the baroclinic destabilization of an axisymmetric rotating gravity current with two incroppings, we derive a number of general theoretical results including necessary conditions for instability (and hence sufficient conditions for stability), a semicircle-like theorem for unstable modes, bounds on the growth rate and phase velocity and conditions for the existence of a high-wavenumber cutoff. The linear solutions as well as the numerical simulations show the preferential amplification of the perturbations to the downslope incropping as available potential energy is released into the instabilities.

The plan of the paper is as follows. The model and geometry are described in $\S 2$. For a simple current configuration in which the height vanishes at two locations in the cross-slope direction, an analytic solution is found, and its stability characteristics are investigated, first for a general height profile in $\S 3$, and then for a specific profile in $\S 4$. In $\S 5$ we compare the predictions of our theory and the experimental data of Lane-Serff \& Baines (1998). Numerical simulations of the fully nonlinear model are presented in $\S 6$.

\section{Governing equations}

Since the model has been derived in detail elsewhere (Swaters 1991), the description here will be brief. The model is derived from two-layer shallow-water theory via an asymptotic expansion in terms of the parameter which measures the ratio of the Nof speed (the speed of a geostrophically balanced density-driven flow on a sloping bottom; see Nof 1983) to the speed of long internal waves, which is assumed to be small. The expansion acts as a low band-pass filter which focuses on the sub-inertial baroclinic dynamics, i.e. filters out internal gravity waves and Kelvin-Helmholtz instabilities.

The non-dimensional equations of the model can be written in the form

$$
\begin{gathered}
\Delta \eta_{t}+J\left(h+\eta, h_{B}\right)+\mu J(\eta, \Delta \eta)=0, \\
h_{t}+J\left(\mu \eta+h_{B}, h\right)=0,
\end{gathered}
$$




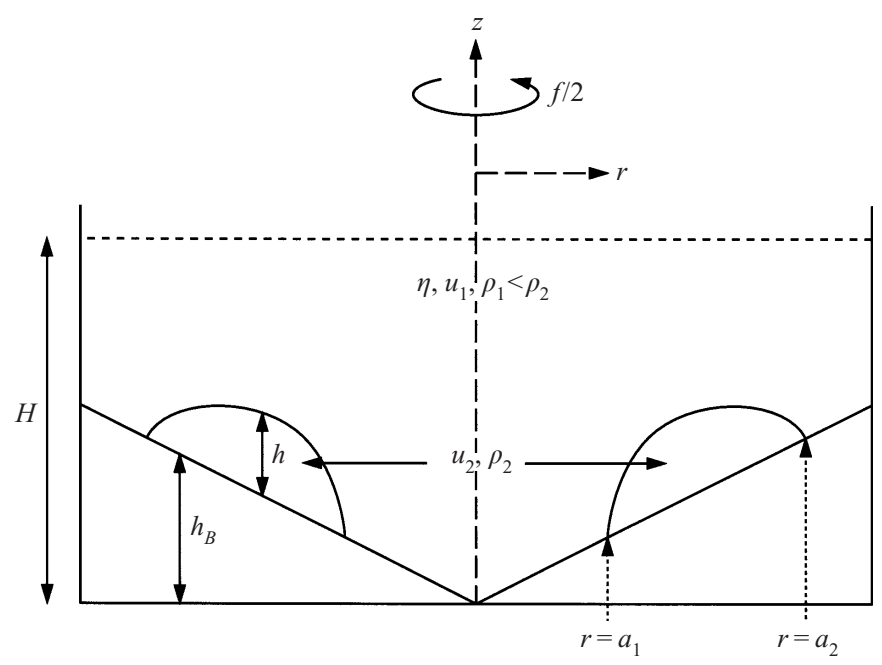

FIGURE 1. The geometry of the model used in this paper.

where $\eta, h$, and $h_{B}$ denote the upper-layer geostrophic pressure, the lower-layer height or thickness, and the bottom topography, respectively (see figure 1). Here, the Jacobian operator $J(A, B)$, denoted as $A_{x} B_{y}-A_{y} B_{x}$ in rectangular coordinates, is for our purposes thought of as representing the vector operation $\hat{\boldsymbol{e}}_{3} \cdot \nabla A \times \nabla B$.

The upper- and lower-layer velocities, $\boldsymbol{u}_{1}$ and $\boldsymbol{u}_{2}$, respectively, and the lower-layer geostrophic pressure, $p$, depend on $\eta$ and $h$ as expressed in the auxiliary equations of the model,

$$
\begin{gathered}
\boldsymbol{u}_{1}=\hat{\boldsymbol{e}}_{3} \times \nabla \eta, \\
\boldsymbol{u}_{2}=\hat{\boldsymbol{e}}_{3} \times \nabla p, \\
p=h_{B}+\mu(\eta+h) .
\end{gathered}
$$

The non-dimensional variables are related to the dimensional (asterisked) variables via the relations

$$
\left.\begin{array}{l}
\left(x^{*}, y^{*}\right)=L(x, y), \quad t^{*}=(s f)^{-1} t, \quad h^{*}=\mu s H h, \\
\boldsymbol{u}_{1}^{*}=\mu s f L \boldsymbol{u}_{1}, \quad \eta^{*}=\mu s(f L)^{2} g^{-1} \eta, \quad \boldsymbol{u}_{2}^{*}=s g^{\prime} H(f L)^{-1} \boldsymbol{u}_{2}, \\
h_{B}^{*}=s H h_{B}, \quad p^{*}=s \rho_{2} g^{\prime} H p,
\end{array}\right\}
$$

where $L=\left(g^{\prime} H\right)^{1 / 2} / f$ is the internal deformation radius of the upper layer, $g^{\prime}$ is the reduced gravity, $H$ is the mean depth of the upper layer, and

$$
s \equiv \frac{h_{B_{*}}}{H}=\frac{s^{*} g^{\prime} / f}{\left(g^{\prime} H\right)^{1 / 2}}
$$

is the aforementioned asymptotic parameter measuring the ratio of the Nof speed to the speed of long internal waves, where $h_{B}$ is the scale height for the bottom topography (precisely, the scale change in topography depth over a distance of $L$ ) and $s^{*}$ the scale slope of the bottom topography. We note that $s$ is identical to the slope Froude number of Maxworthy (1997). Also, we observe that the parameter 
regime in which Etling \& Chabert d'Hieres (1997) observe strong eddy formation in the lower-layer current (high rotation rates, low slope, low density excess and a weak source rate) is exactly the parameter regime necessary to ensure that $s$ is small.

The interaction parameter $\mu$ is given by

$$
\mu=\frac{h_{*}}{h_{B_{*}}},
$$

where $h_{*}$ is the scale height of the gravity current. The interaction parameter provides a measure of the ratio of the destabilizing effect of baroclinic vortex tube stretching to the stabilizing effect of the bottom topography, which acts like a topographic $\beta$-plane. Previous studies of this model in various contexts (Swaters 1991; Karsten et al. 1995) have found that the baroclinic instability predicted by this model depends strongly upon the interaction parameter, and we expect a similar dependence here.

Typical values for $\mu$ are $O(1)$. In geophysical contexts, Swaters (1991) estimates $\mu \approx 2$ for the cold pool observed travelling on the New England Bight by Houghton et al. (1982), and Karsten et al. (1995) find that $\mu \approx 1$ is appropriate for the deep-water replacement current in the Strait of Georgia. These values are readily reproduced in the laboratory, e.g. Lane-Serff \& Baines (1998) generate rotating tank flows on a sloping bottom with approximately $0.1 \leqslant \mu \leqslant 10$ (see their figure $12 b$ ).

It is important to note that, by focusing on subinertial baroclinic dynamics as the driving physical process behind the instability of these currents, other dynamical processes such as higher-frequency effects, barotropic instability, lateral and bottom friction, mixing, and thermodynamic interactions between the layers are neglected. Of these processes, the effects of friction may be the most significant, particularly in laboratory flows. It is therefore necessary to briefly consider the observed effects of friction on these flows so as to carefully identify the circumstances under which a frictionless model is appropriate.

On a coupled-front bottom-dwelling current, friction acts to broaden the current, move it in the downslope direction, and decrease its height, due to loss of mass through Ekman pumping (Condie 1995). Laboratory flows are easily generated where viscous effects are small enough that the flow is unstable, and breaks up into a train of eddies. Typically, the movement of the eddies seems decoupled from the viscous flow, the former travelling along an isobath, and the latter forming a thin Ekman layer which flows with a significant downslope component to its velocity (Smith 1977; Whitehead et al. 1990; Lane-Serff \& Baines 1998). Viscous drag on the eddies can cause them to have a small downslope drift as well, although they have been observed to have an upslope drift resulting from Ekman draining and upper-layer potential vorticity conservation (Mory et al. 1987; Whitehead et al. 1990). The viscous flow has been observed to develop roll waves in certain parameter regimes (Whitehead et al. 1990; Nagata et al. 1993; Zatsepin, Kostyanoi \& Semenov 1996; Lane-Serff \& Baines 1998).

We take advantage of the observed decoupling of the viscous and inviscid flow, and determine the instability characteristics of the inviscid part. This means that, in the laboratory, our analysis applies to flows that are significantly thicker than the Ekman layer thickness, and for timescales on which Ekman draining does not remove a significant fraction of the lower-layer mass.

We wish to focus on a cylindrical geometrical configuration. Let $r$ be the radial coordinate and $\theta$ the azimuthal coordinate. Assuming $h_{B}=h_{B}(r)$, it follows from (2.1) 
and (2.2) that

$$
\begin{gathered}
\Delta \eta_{t}-\frac{1}{r} \frac{\partial h_{B}}{\partial r}\left(\frac{\partial h}{\partial \theta}+\frac{\partial \eta}{\partial \theta}\right)+\mu J(\eta, \Delta \eta)=0, \\
h_{t}+\frac{1}{r} \frac{\partial h_{B}}{\partial r} \frac{\partial h}{\partial \theta}+\mu J(\eta, h)=0,
\end{gathered}
$$

where $J(A, B)=\left(A_{r} B_{\theta}-A_{\theta} B_{r}\right) / r$.

We are particularly interested in determining the evolution of the perturbed incroppings, i.e. the location where the lower-layer height vanishes. If we project the location of the intersection of the lower layer with the bottom topography onto a horizontal plane and denote this function as $\phi(r, \theta, t)=0$, then the kinematic condition gives

$$
\frac{\partial \phi}{\partial t}+\frac{1}{r} \frac{\partial h_{B}}{\partial r} \frac{\partial \phi}{\partial \theta}+\mu J(\eta+h, \phi)=0,
$$

evaluated on $\phi=0$, and the dynamic boundary condition is simply $h(\phi=0)=0$.

For boundary conditions, we require no normal flow on the tank walls. In addition, since we are working in a cylindrical geometry, periodicity with respect to $\theta$ must be satisfied. These conditions may be expressed as

$$
\begin{gathered}
\frac{\partial \eta}{\partial \theta}=0 \quad \text { on } r=r_{\max }, \\
\eta(r, \theta+2 \pi, t)=\eta(r, \theta, t),
\end{gathered}
$$

where $r_{\max }$ is the tank radius.

\section{General linear results}

It is straightforward to verify that $\eta=\eta_{0}(r), h=h_{0}(r)$ is an exact solution to the nonlinear equations. In order to focus on baroclinic instability (i.e. we are excluding any possible barotropic instability in the upper layer), we choose to linearize about the solution where $\eta_{0}=0$. We study the stability of a current whose height vanishes at two different values of $r$, so as to produce an annulus-shape flow with two incroppings (see figure 1). We thus assume the following form:

$$
\begin{gathered}
h=h_{0}(r)+h^{\prime}(r, \theta, t) \quad \text { for } a_{1}<r<a_{2}, \\
\eta=\eta^{\prime}(r, \theta, t) \quad \text { for } 0<r<r_{\max }, \\
\phi=\left\{\begin{array}{l}
\phi_{1}=r-a_{1}+\phi_{1}^{\prime}(\theta, t) \\
\phi_{2}=r-a_{2}+\phi_{2}^{\prime}(\theta, t),
\end{array}\right.
\end{gathered}
$$

i.e. $h_{0}(r)>0$ for $r \in\left(a_{1}, a_{2}\right)$ and $h_{0}(r) \equiv 0$ for $r \in\left[0, a_{1}\right] \cup\left[a_{2}, r_{\max }\right]$.

Upon substituting the above into (2.7), (2.8), and (2.9), and dropping the primes, the linearized stability equations become

$$
\begin{gathered}
\Delta \eta_{t}-h_{B_{r}}\left(\eta_{\theta}+h_{\theta}\right) / r=0, \\
h_{t}+\left(h_{B_{r}} h_{\theta}-\mu h_{0_{r}} \eta_{\theta}\right) / r=0,
\end{gathered}
$$

where the subscripts denote partial differentiation. We assume azimuthally-propagating 
normal mode solutions of the form

$$
\left[\eta, h, \phi_{1}, \phi_{2}\right]=\left[\tilde{\eta}(r), \tilde{h}(r), \tilde{\phi}_{1}, \tilde{\phi}_{2}\right] \exp [\operatorname{in}(\theta-c t)]+\text { c.c. },
$$

where c.c. stands for the complex conjugate of the preceding expression, and the wavenumber is written as $n$ to emphasize that it must take integer values to satisfy the periodicity condition. Note that, dimensionally, $n$ and $c$, the complex-valued phase velocity, have units of $\operatorname{rad}^{-1}$ and $\mathrm{rad} \mathrm{s}^{-1}$, respectively.

The normal mode problem, after dropping the tildes, is

$$
\left.\begin{array}{c}
\frac{\mathrm{d}}{\mathrm{d} r}\left(r \frac{\mathrm{d}}{\mathrm{d} r} \eta\right)-\frac{n^{2}}{r} \eta+h_{B}^{\prime}\left(\frac{1}{c}-\frac{\mu}{c^{2}} \frac{h_{0}^{\prime}(r)}{\left(r-h_{B}^{\prime} / c\right)}\right) \eta=0 \\
h=-\frac{\mu}{c} \frac{h_{0}^{\prime}(r)}{\left(r-h_{B}^{\prime} / c\right)} \eta
\end{array}\right\} \text { for } a_{1}<r<a_{2},
$$

where $h_{0}^{\prime}=h_{0_{r}}$ and $h_{B}^{\prime}=h_{B_{r}}$. Boundary condition (2.10) for the normal mode problem becomes

$$
\eta\left(r_{\max }\right)=0
$$

and it is understood, of course, that $\eta(0)$ must be bounded. The other conditions required are the jump conditions at the incropping locations $r=a_{1,2}$. The continuity of normal mass flux across $r=a_{1,2}$ implies that $\eta$ must be continuous across $r=a_{1,2}$. It follows from integrating (3.4) across $r=a_{1,2}$, even if $h_{0}^{\prime}(r)$ is discontinuous there (but remains bounded), that the jump in $\eta_{r}$ must be zero. Hence the appropriate matching conditions on $\eta$ across $r=a_{1,2}$ are that it be continuously differentiable.

\subsection{Semicircle theorem}

We may derive a theorem analogous to Howard's semicircle theorem (Pedlosky 1987) for the locations in complex phase velocity space of unstable modes. Equations (3.4) and (3.5) may be written as

$$
\frac{\mathrm{d}}{\mathrm{d} r}\left(r \frac{\mathrm{d}}{\mathrm{d} r} \eta\right)-\frac{n^{2}}{r} \eta+\frac{h_{B}^{\prime}}{c}\left(1+\frac{\mu \Theta(r) h_{0}^{\prime}(r)}{\left(h_{B}^{\prime}-c r\right)}\right) \eta=0,
$$

where $\Theta(r)$ takes the value of unity when $a_{1}<r<a_{2}$ (i.e. (3.4) applies), and it equals zero otherwise (i.e. (3.5) applies). If (3.7) is multiplied by $\eta^{*}$, the complex conjugate of $\eta$, and integrated from $r=0$ to $r=r_{\max }$, then, assuming instability, where $c=c_{R}+\mathrm{i} c_{I}$, the real and imaginary parts of the resulting expression are, respectively,

$$
\begin{aligned}
c_{R}\left\{\int_{0}^{r_{\max }}\left[r\left|\eta_{r}\right|^{2}+\left(\frac{n^{2}}{r}+\frac{r}{\left|h_{B}^{\prime}-c r\right|^{2}} \mu h_{B}^{\prime} h_{0}^{\prime} \Theta(r)\right)|\eta|^{2}\right] \mathrm{d} r\right\} \\
=\int_{0}^{r_{\max }}\left(1+\frac{\mu h_{B}^{\prime} h_{0}^{\prime} \Theta(r)}{\left|h_{B}^{\prime}-c r\right|^{2}}\right) h_{B}^{\prime}|\eta|^{2} \mathrm{~d} r,
\end{aligned}
$$

and

$$
c_{I}\left\{\int_{0}^{r_{\max }}\left[r\left|\eta_{r}\right|^{2}+\left(\frac{n^{2}}{r}-\frac{r}{\left|h_{B}^{\prime}-c r\right|^{2}} \mu h_{B}^{\prime} h_{0}^{\prime} \Theta(r)\right)|\eta|^{2}\right] \mathrm{d} r\right\}=0 .
$$


If a mode is unstable, then $c_{I} \neq 0$, and so the integral in (3.9) must vanish. Thus

$$
\int_{a_{1}}^{a_{2}} \frac{r h_{B}^{\prime} h_{0}^{\prime}}{\left|h_{B}^{\prime}-c r\right|^{2}}|\eta|^{2} \mathrm{~d} r=Q / \mu,
$$

where

$$
Q \equiv \int_{0}^{r_{\max }}\left\{r\left|\eta_{r}\right|^{2}+\frac{n^{2}}{r}|\eta|^{2}\right\} \mathrm{d} r>0 .
$$

Therefore, a necessary condition for instability is that $h_{B}^{\prime} h_{0}^{\prime}>0$ somewhere in the flow. If we examine figure 1 , in which $h_{B}^{\prime}$ is a positive constant, we see that a lowerlayer height profile shaped like a coupled front will satisfy the necessary condition for instability on the downslope side but not on the upslope side. This observation underscores the asymmetrical cross-front structure of the unstable modes which we will see.

Assuming instability, we may therefore define

$$
\gamma^{2} \equiv \max _{a_{1} \leqslant r \leqslant a_{2}} h_{B}^{\prime} h_{0}^{\prime} .
$$

Then (3.10) gives

$$
\frac{\gamma^{2}}{a_{1}} \int_{a_{1}}^{a_{2}} \frac{|\eta|^{2} \mathrm{~d} r}{\left|c-h_{B}^{\prime} / r\right|^{2}} \geqslant \frac{Q}{\mu},
$$

which, upon rearrangement yields

$$
\min _{\left(a_{1}, a_{2}\right)}\left|c-h_{B}^{\prime} / r\right|^{2} \leqslant \frac{\mu \gamma^{2}}{Q a_{1}} \int_{a_{1}}^{a_{2}}|\eta|^{2} \mathrm{~d} r .
$$

In the usual derivation of a semicircle theorem on a $\beta$-plane (e.g. Pedlosky 1987), a Poincaré Inequality is invoked to find an upper bound for the right-hand side. However, we do not need one here. We observe that

$$
\begin{aligned}
Q= & \int_{0}^{r_{\max }}\left\{r\left|\eta_{r}\right|^{2}+\frac{n^{2}}{r}|\eta|^{2}\right\} \mathrm{d} r \\
& \geqslant \int_{a_{1}}^{a_{2}}\left(n^{2}|\eta|^{2} / r\right) \mathrm{d} r \\
& \geqslant \frac{n^{2}}{a_{2}} \int_{a_{1}}^{a_{2}}|\eta|^{2} \mathrm{~d} r .
\end{aligned}
$$

Upon substitution of (3.14) into (3.13), one finds

$$
\min _{\left(a_{1}, a_{2}\right)}\left|c-h_{B}^{\prime} / r\right|^{2} \leqslant \mu \gamma^{2} a_{2}\left(n^{2} a_{1}\right)^{-1} .
$$

Explicitly, this says that if instability occurs, then the complex phase velocity $c$ must lie in the region

$$
\left.\begin{array}{ll}
\left(c_{R}-c_{1}\right)^{2}+c_{I}^{2} \leqslant \mu \gamma^{2} a_{2}\left(n^{2} a_{1}\right)^{-1} & \text { if } c_{R}<c_{1}, \\
c_{I} \leqslant \gamma\left(\mu a_{2} / a_{1}\right)^{1 / 2} n^{-1} & \text { if } c_{1} \leqslant c_{R} \leqslant c_{2}, \\
\left(c_{R}-c_{2}\right)^{2}+c_{I}^{2} \leqslant \mu \gamma^{2} a_{2}\left(n^{2} a_{1}\right)^{-1} & \text { if } c_{R}>c_{2},
\end{array}\right\}
$$


where $c_{1}=\min _{a_{1}<r<a_{2}}\left(h_{B}^{\prime} / r\right)$ and $c_{2}=\max _{a_{1}<r<a_{2}}\left(h_{B}^{\prime} / r\right)$. It follows immediately from (3.16) that the maximum growth rate possible is

$$
\sigma=n c_{I} \leqslant \gamma\left(\mu a_{2} / a_{1}\right)^{1 / 2} .
$$

\subsection{Other stability results}

The semicircle theorem provides bounds on the phase velocity for an unstable mode. By deriving an alternative set of bounds on the real part of the phase velocity, we may demonstrate the existence of a high-wavenumber cutoff for the existence of unstable modes. Upon substituting (3.10) and (3.11) into (3.8), one finds

$$
c_{R}=(2 Q)^{-1} \int_{0}^{r_{\max }}\left(1+\frac{\mu h_{B}^{\prime} h_{0}^{\prime} \Theta(r)}{\left|h_{B}^{\prime}-c r\right|^{2}}\right) h_{B}^{\prime}|\eta|^{2} \mathrm{~d} r,
$$

from which, upon employing (3.10) and a similar set of arguments to the one which led to (3.14), one obtains bounds on the real part of the phase velocity

$$
c_{1} / 2 \leqslant c_{R} \leqslant c_{2} / 2+\max _{\left(0, r_{\max }\right)}\left(r h_{B}^{\prime}\right) /\left(2 n^{2}\right) .
$$

However, the semicircle theorem already limits the real part of the phase velocity for an unstable mode,

$$
c_{1}-\gamma\left(\mu a_{2} / a_{1}\right)^{1 / 2} n^{-1} \leqslant c_{R} \leqslant c_{2}+\gamma\left(\mu a_{2} / a_{1}\right)^{1 / 2} n^{-1} .
$$

The relations (3.19) and (3.20) hold even if $h_{B}^{\prime}<0$, in which case $c_{R}<0$. When $h_{B}^{\prime}>0$ for all $r$, the requirement that (3.19) and (3.20) hold simultaneously means that the correct range of $c_{R}$, for sufficiently large $n$, is

$$
c_{1}-\gamma\left(\mu a_{2} / a_{1}\right)^{1 / 2} / n \leqslant c_{R} \leqslant c_{2} / 2+\max _{\left(0, r_{\max }\right)}\left(r h_{B}^{\prime}\right) /\left(2 n^{2}\right) .
$$

When $h_{B}^{\prime}<0$ for all $r$, the correct range is

$$
\min _{\left(a_{1}, a_{2}\right)}\left(h_{B}^{\prime} / r\right) / 2 \leqslant c_{R} \leqslant \max _{\left(a_{1}, a_{2}\right)}\left(h_{B}^{\prime} / r\right)+\gamma\left(\mu a_{2} / a_{1}\right)^{1 / 2} / n .
$$

However, upon multiplying through by -1 , one finds that this is equivalent to

$$
\min _{\left(a_{1}, a_{2}\right)}\left(\left|h_{B}^{\prime}\right| / r\right)-\gamma\left(\mu a_{2} / a_{1}\right)^{1 / 2} / n \leqslant\left|c_{R}\right| \leqslant \max _{\left(a_{1}, a_{2}\right)}\left(\left|h_{B}^{\prime}\right| / r\right) / 2,
$$

which is a range of $\left|c_{R}\right|$ contained within (3.21). Thus, we redefine $c_{1}=$ $\min _{a_{1}<r<a_{2}}\left(\left|h_{B}^{\prime}\right| / r\right)$ and $c_{2}=\max _{a_{1}<r<a_{2}}\left(\left|h_{B}^{\prime}\right| / r\right)$, so that (3.21) is true as stated for $h_{B}^{\prime}>0$ and $h_{B}^{\prime}<0$.

So for an unstable mode to exist, it must necessarily be true that

$$
c_{1}-\gamma\left(\mu a_{2} / a_{1}\right)^{1 / 2} / n \leqslant c_{2} / 2+\max _{\left(0, r_{\max }\right)}\left(r\left|h_{B}^{\prime}\right|\right) /\left(2 n^{2}\right) .
$$

We can use this relation to show the existence of a high-wavenumber cutoff for unstable modes. However, the above will hold true for all sufficiently large $n$ if $c_{1} \leqslant c_{2} / 2$. Thus for the purposes of this proof, we assume the contrary, i.e. that

$$
c_{2}<2 c_{1} \text {. }
$$

Physically, this requirement says that the current must be sufficiently narrow with respect to its distance from the origin, e.g. with a linearly sloping bottom, (3.23) reduces to $a_{2}<2 a_{1}$. Clearly, this requirement would have no analogy in the problem studied in a rectangular geometry (see Swaters 1991), since that problem may be 
thought of as a limiting case of this one where the radius of the tank goes to infinity, while the distance of the current from the edge of the tank is held fixed, i.e. the problem studied by Swaters (1991) is recovered in the limit as $a_{1} \rightarrow \infty$ with $a_{2}-a_{1}$ and $r_{\max }-a_{2}$ held fixed. We cannot apply these arguments to the experiments described by Griffiths et al. (1982) since they were of surface currents over a flat bottom, so essential aspects of the theory we develop here are missing. However, as evidence that (3.23) is satisfied by reasonable laboratory parameters, we observe that each one of the annulus currents reported by Griffiths et al. (1982) do indeed satisfy $a_{2}<2 a_{1}$.

Assuming (3.23), we may rearrange (3.22) into a quadratic inequality in $n$, from which the quadratic formula yields

$$
n \leqslant n_{\max }=\frac{\gamma\left(\mu a_{2} a_{1}^{-1}\right)^{1 / 2}+\left[\gamma^{2} \mu a_{2} a_{1}^{-1}+\left(\max _{\left(0, r_{\max }\right)}\left(r\left|h_{B}^{\prime}\right|\right)\right)\left(2 c_{1}-c_{2}\right)\right]^{1 / 2}}{2 c_{1}-c_{2}},
$$

which establishes a high-wavenumber cutoff for unstable modes, i.e. any mode with wavenumber greater than $n_{\max }$ is stable.

We emphasize that (3.23) is merely a sufficient condition, not a necessary one, for the existence of a high-wavenumber cutoff. We conjecture that a high-wavenumber cutoff exists even when (3.23) is violated. This is consistent with the numerical solutions of the dispersion relation we present in the next section.

Maintaining assumption (3.23), we may derive a lower bound for the interaction parameter $\mu$ required for instability (for a given $n$ ). Equation (3.22) may be solved for $\mu$ to find that if

$$
c_{1}-c_{2} / 2-\max _{\left(0, r_{\max }\right)}\left(r\left|h_{B}^{\prime}\right|\right) /\left(2 n^{2}\right)>0,
$$

then the existence of an unstable mode requires that

$$
\mu \geqslant \mu_{\text {min }}=\frac{n^{2} a_{1}}{\gamma^{2} a_{2}}\left[c_{1}-c_{2} / 2-\max _{\left(0, r_{\text {max }}\right)}\left(r\left|h_{B}^{\prime}\right|\right) /\left(2 n^{2}\right)\right]^{2} .
$$

Since the parameter $\mu=h_{*}\left(s^{*} L\right)^{-1}$, where $h_{*}, s^{*}$ and $L$ are, respectively, the maximum height of the gravity current, the slope of the bottom topography and the horizontal length scale which is the internal deformation radius based on the mean thickness of the upper layer, $\mu_{\min }$ can be interpreted as requiring a minimum current height (for a given bottom slope and stratification) for baroclinic instability. It would be interesting to test this prediction against laboratory experiments.

\section{Solution for a parabolic height profile}

We investigate the linear stability problem for the case where the bottom topography is conical, i.e. $h_{B}=r$ (see figure 1), and where the lower-layer height profile is specified to be parabolic in the radial direction with two incroppings. Let

$$
h_{0}(r)=1-\left(r-r_{0}\right)^{2} / a^{2},
$$

where the new parameters, $r_{0}$ and $a$ denote the radius of maximum height of the current $\left(h_{0}\left(r_{0}\right)=1\right)$ and the half-width of the current, respectively. To relate these to the parameters defined previously, the incropping locations are given by $a_{1}=r_{0}-a$ and $a_{2}=r_{0}+a$. Note that this initial shape for the bottom current indeed satisfies the necessary condition for instability that there exist $r$-values for which $h_{0_{r}}>0$, and that 
this occurs on the side of the current closest to the axis of rotation. For this current to satisfy the sufficient condition (3.23) for the existence of a high-wavenumber cutoff, we require that $a<r_{0} / 3$.

For $h_{0}$ given by (4.1), (3.4) and (3.5) become

$$
\begin{array}{cc}
\eta^{\prime \prime}+\frac{1}{r} \eta^{\prime}+\left(\frac{1}{c r}-\frac{n^{2}}{r^{2}}+\frac{2 \mu}{c^{2} a^{2}} \frac{r-r_{0}}{r(r-1 / c)}\right) \eta=0 & \text { for } r_{0}-a<r<r_{0}+a, \\
\eta^{\prime \prime}+\frac{1}{r} \eta^{\prime}+\left(\frac{1}{c r}-\frac{n^{2}}{r^{2}}\right) \eta=0 & \text { elsewhere, }
\end{array}
$$

where $(*)^{\prime}=(*)_{r}$. Equation (4.3) has the closed-form solution

$$
\eta=A J_{2 n}\left(2 c^{-1 / 2} r^{1 / 2}\right)+B Y_{2 n}\left(2 c^{-1 / 2} r^{1 / 2}\right),
$$

where $J$ and $Y$ denote Bessel and Neumann functions, respectively, and $A$ and $B$ are constant coefficients which must be determined from the boundary conditions. $A$ and $B$ will, of course, be different in each of the two regions $0<r<r_{0}-a$ and $r_{0}+a<r<r_{\max }$.

A Frobenius solution to (4.2) can be constructed, but there are some mathematical technicalities which need to be considered. Note that (4.2) has two regular singular points: one at $r=0$, and another at $r=1 / c$. The expansion is required to converge for all $r_{0}-a<r<r_{0}+a$. Because of the existence of both regular singular points, no single complex $r$ can be chosen such that the expansion about that point will converge for all real $r \in\left(r_{0}-a, r_{0}+a\right)$. For example, an expansion about $r=1 / c$ will fail to converge at $r=r_{0}$ if $|1 / c|<r_{0} / 2$, but an expansion about $r=r_{0}$ will fail to converge at $r=r_{0}+a$ if $\left|r_{0}-1 / c\right|<a$. Thus, for any given complex $c$, a Frobenius expansion is performed about the regular singular point $r=1 / c$ if $\left|r_{0}-1 / c\right| \leqslant a$, and the expansion is performed about the regular point $r=r_{0}$ if $\left|r_{0}-1 / c\right|>a$.

In this way, two linearly independent solutions to (4.2) can always be constructed. Let $\eta_{1}$ and $\eta_{2}$ denote these two solutions. Since we are required to calculate $\eta_{1}$ and $\eta_{2}$ for various complex values of $c$ in order to determine the solution to the dispersion relation, care must be exercised to ensure $\eta_{1}$ and $\eta_{2}$ are consistently defined for the different expansions. For either expansion, we define $\eta_{1}$ and $\eta_{2}$ such that

$$
\eta_{1}\left(r_{0}\right)=1, \quad \eta_{1}^{\prime}\left(r_{0}\right)=0, \quad \eta_{2}\left(r_{0}\right)=0, \quad \eta_{2}^{\prime}\left(r_{0}\right)=1 .
$$

Thus the solution to (4.2) and (4.3) may be written in the form

$$
\eta(r)= \begin{cases}A J_{2 n}\left(2 r^{1 / 2} c^{-1 / 2}\right) & \text { for } 0 \leqslant r \leqslant r_{0}-a \\ B \eta_{1}(r)+C \eta_{2}(r) & \text { for } r_{0}-a<r<r_{0}+a \\ D J_{2 n}\left(2 r^{1 / 2} c^{-1 / 2}\right)+E Y_{2 n}\left(2 r^{1 / 2} c^{-1 / 2}\right) & \text { for } r_{0}+a \leqslant r \leqslant r_{\max },\end{cases}
$$

where $A, B, C, D$ and $E$ are arbitrary constants and we have employed the condition that $\eta$ be bounded at the origin.

The dispersion relation is obtained by applying the jump conditions

$$
[\eta]=0 \quad \text { and } \quad\left[\eta^{\prime}\right]=0 \quad \text { at } r=r_{0} \pm a,
$$

and the remaining boundary condition $\eta\left(r_{\max }\right)=0$ to the solution (4.6). This produces five equations in the five unknown arbitrary constants. The necessary condition that there exists a non-trivial solution to these homogeneous equations is that the determinant of the matrix of their coefficients vanishes. This requirement determines 

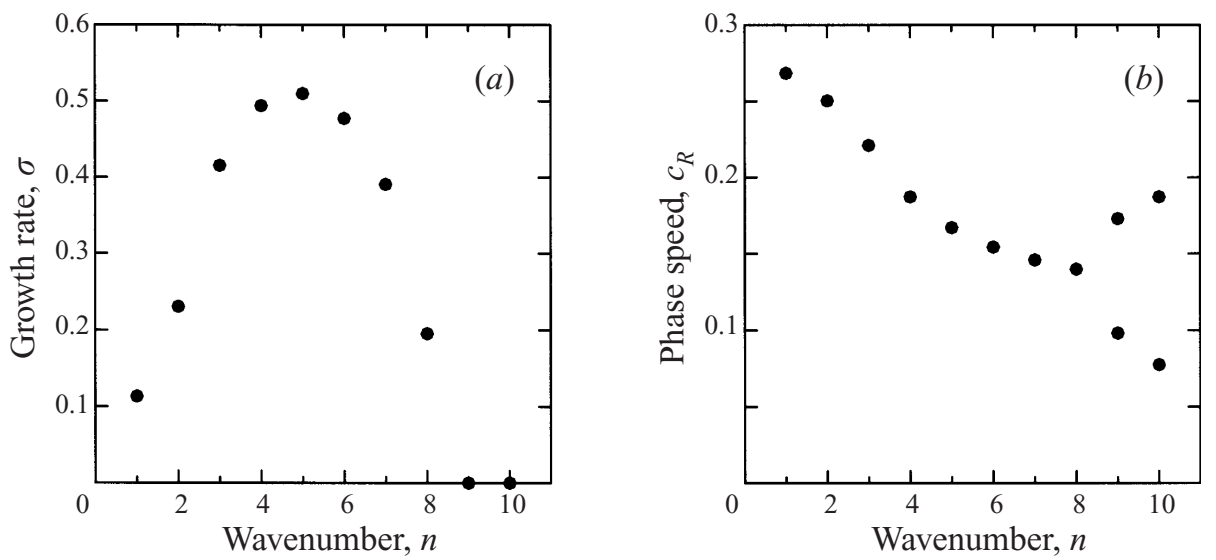

FIGURE 2 . The dispersion relation with parameters $\mu=1, r_{\max }=2 \pi, a=0.75$ and $r_{0}=2 \pi-2 a$. Modes not shown with $10<n \leqslant n_{\max }=27$ are stable.
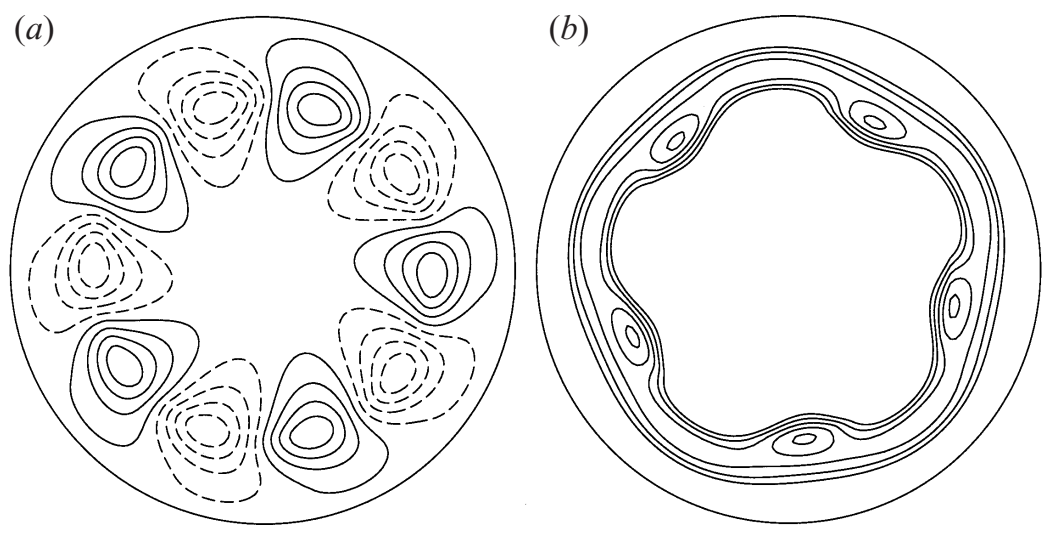

FigURE 3. The spatial form of the most unstable mode shown in figure $2(n=5)$. Parameters are $\mu=1, r_{\max }=2 \pi, a=0.75$ and $r_{0}=2 \pi-2 a$. (a) Upper-layer stream function or geostrophic pressure (the contour interval is 0.066); $(b)$ lower-layer height (the contour interval is 0.3 ). Dotted lines indicate negative values.

the dispersion relation, which we write formally as

$$
c=c\left(\mu, a, r_{\max }, n, r_{0}\right),
$$

and which we solve numerically.

The dispersion relation is shown in figure 2, where we plot the growth rate, $\sigma=n\left|c_{I}\right|$, and the angular phase speed, $c_{R}$, versus wavenumber for parameter values $\mu=1$, $a=0.75, r_{\max }=2 \pi$ and $r_{0}=2 \pi-2 a$. Besides the discrete wavenumber required to satisfy periodicity conditions, the plots match qualitatively the analogous plots found by Swaters (1991) and Karsten et al. (1995), each of whom used similar models, but in different geometries and applications. The matching features include the fact that the growth rate goes linearly to zero as the wavenumber approaches zero, the existence of a maximum growth rate at a particular wavenumber, and the existence of a high-wavenumber cutoff.

According to figure 2, the most unstable wavenumber is $n=5$. To relate this 

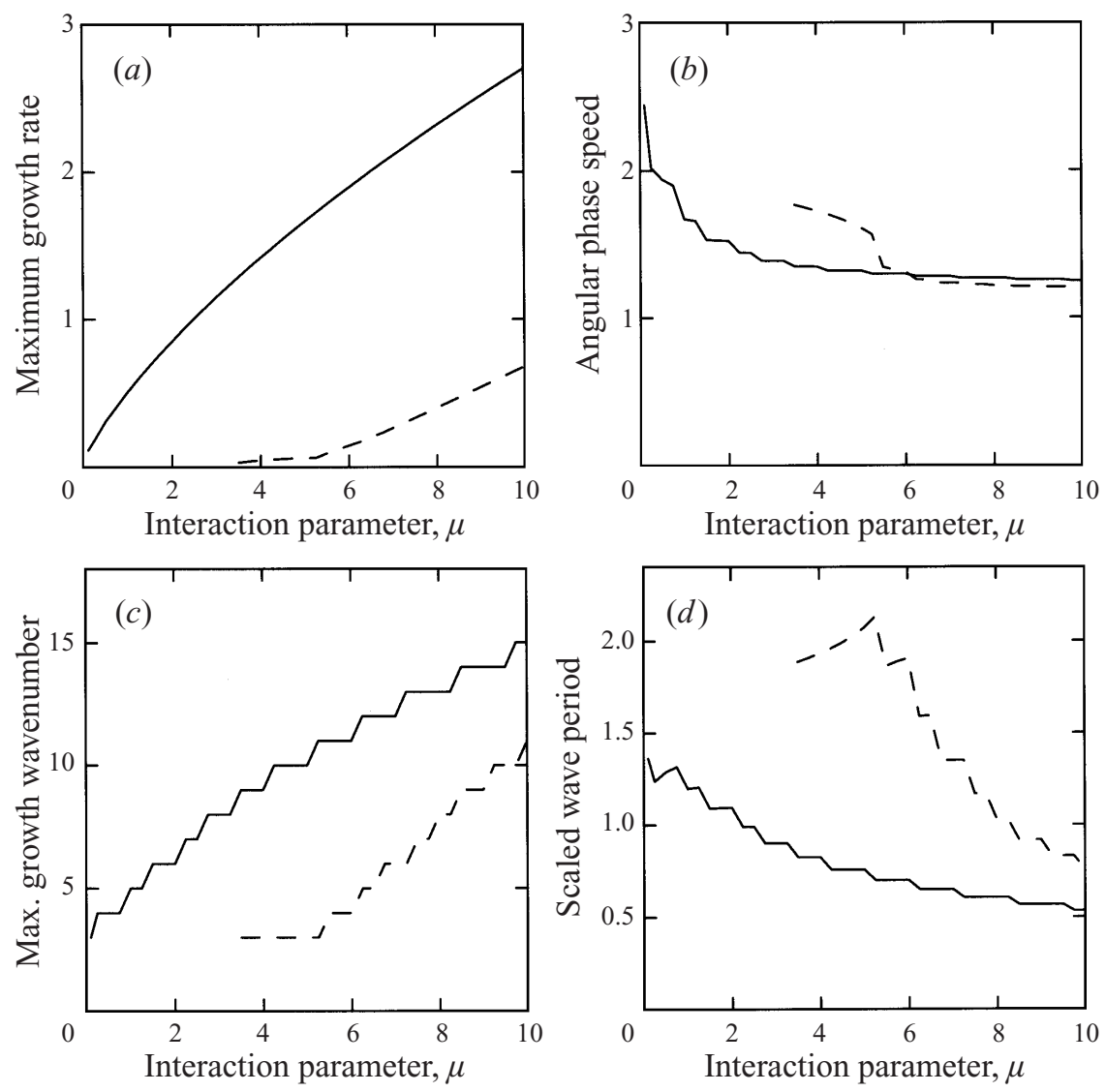

FIGURE 4. Dependence of disturbance characteristics upon interaction parameter $\mu$. Other parameter values are $r_{\max }=2 \pi, a=0.75$ and $r_{0}=2 \pi-2 a$. Solid line represents the monopole mode, dashed line represents the dipole mode. Wavenumber discretization is the cause of the jagged appearance of some of the plotted lines.

to dimensional variables, the wavelength of the fastest growing wave (as measured around the circle of radius $r_{0}$ ) is $2 \pi r_{0} L / 5 \approx 6.0 L$ for the parameters used in figure 2. Assuming typical laboratory parameters of $H=20 \mathrm{~cm}, g^{\prime}=2.5 \mathrm{~cm} \mathrm{~s}^{-2}$, and $f=$ $1.0 \mathrm{~s}^{-1}$, one finds $L \approx 7 \mathrm{~cm}$, so that the wavelength of the fastest growing wave is then $42 \mathrm{~cm}$.

When $c_{I}=0$, then the point $r_{c}=1 / c$ will be a critical point provided $a_{1}<r_{c}<a_{2}$. This occurs for one point shown in figure 2, on the upper branch of the neutral curve at $n=10$. Despite the presence of the critical $r$ value, the $\eta$ and $h$ fields corresponding to that point are well behaved; $\eta(r)$ is observed to pass through zero linearly with $(r-1 / c)$, so that $h(r) \propto \eta(r) /(r-1 / c)$ is $O(1)$ there, as are the other physical quantities. We have run a numerical simulation of the fully nonlinear model, using the numerical procedure described in $\S 6$, which was initialized with fields corresponding to this point on the neutral curve, and we see no evidence of the critical point affecting the solution.

Figure 3 displays the spatial form of the most unstable mode $(n=5)$ for the same set of parameters as in figure 2 (i.e. $\mu=1, a=0.75, r_{\max }=2 \pi$ and $r_{0}=2 \pi-2 a$ ). Note that the lower layer is deformed on the downward side more than on the 

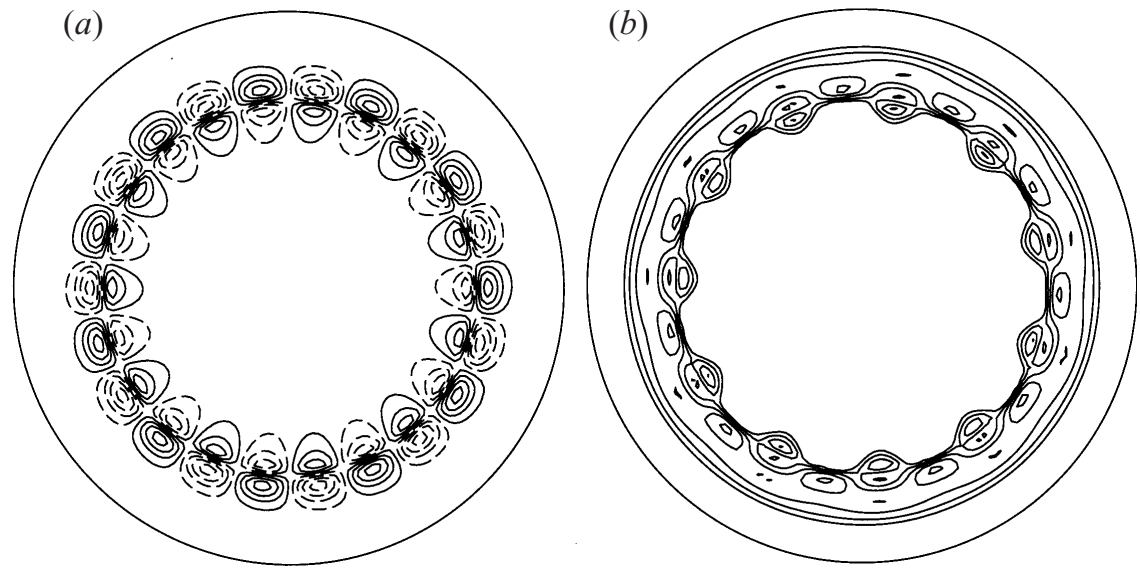

FIGURE 5. The spatial form of the most unstable dipole mode at $\mu=10(n=11)$. Parameters are $r_{\max }=2 \pi, a=0.75$ and $r_{0}=2 \pi-2 a$. (a) Upper-layer stream function or geostrophic pressure (the contour interval is 0.0066); (b) lower-layer height (the contour interval is 0.26 ). Dotted lines indicate negative values.
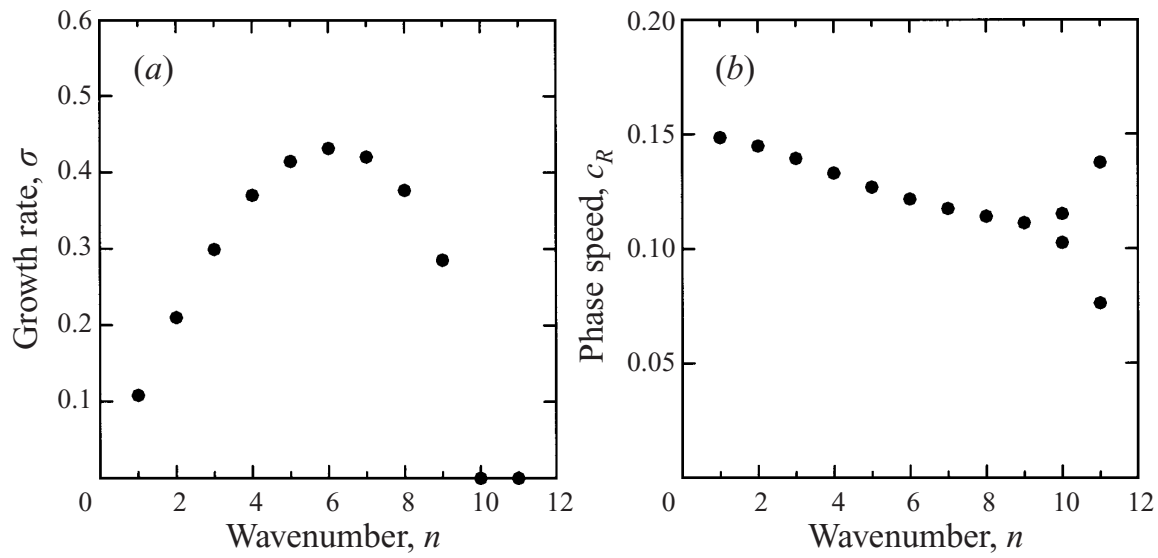

FiguRE 6. Dispersion relation for $h_{B}^{\prime}=-1$, and all other parameters exactly as in figure 2. Modes not shown are stable.

upward side. This is a result of the lower layer losing potential energy to fuel the instability.

The normal mode solutions become more unstable as the interaction parameter $\mu$ increases. Figure 4 shows the maximum growth rate $\sigma$ versus $\mu$ where the remaining parameters have the values $a=0.75, r_{\max }=2 \pi$ and $r_{0}=2 \pi-2 a$. Also plotted are the phase speed $c_{R}$, wavenumber $n$ and scaled wave period $1 /\left(c_{R} n\right)$ corresponding to the maximum growth rate. The scaled wave period is a quantity that may readily be compared with experimental data, which we will do in $\S 5$. The maximum growth rate and corresponding wavenumber display a strong dependence on $\mu$. Note that a new unstable mode exists for $\mu>3.5$. This mode is the dipole mode, whose spatial form is shown in figure 5 for $\mu=10, a=0.75, r_{\max }=2 \pi$ and $r_{0}=2 \pi-2 a$.

Since many rotating tank experiments have been performed with axisymmetric bottom topography which slopes away from the centre (e.g. Smith 1977; Nagata et al. 1993; Zatsepin et al. 1996, 1998; Lane-Serff \& Baines 1998), we show in figure 6 

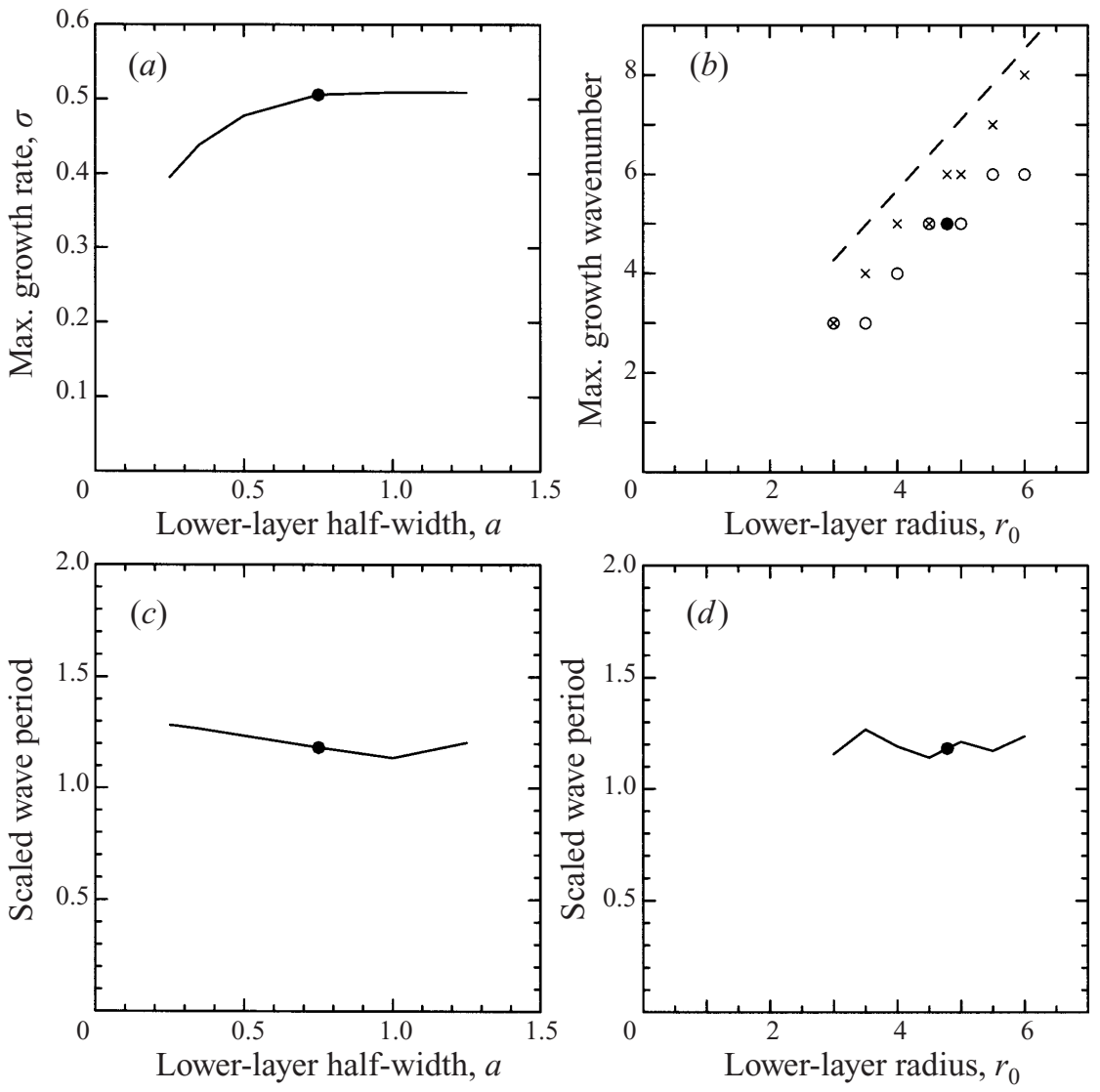

FIGURE 7. Other parameter dependences. In each plot, the filled circle corresponds to the reference case shown in figure 2. (a) Maximum growth rate versus half-width of the lower layer. (b) The dependence of the wavenumber of maximum growth on the mean radius of the lower layer $r_{0}$. Open circles are for parameters $\mu=1, a=0.75$ and $r_{\max }=7.5$, for comparison with figure 2 , with the filled circle at $r_{0}=2 \pi-2 a$. $\times$ symbols are for parameters $\mu=2, a=1.0$ and $r_{\max }=7.5$, for comparison with Swaters (1991), represented by the dashed line $n=1.421 r_{0}$. The $\times$ symbols falling below the dashed line is an indication that the instability takes place on the downslope side of the current. $(c, d)$ Dependence of the scaled wave period, $1 /\left(c_{R} n\right)$ where $c_{R}$ and $n$ are for the fastest-growing mode, on $a$ and $r_{0}$ respectively. The lack of strong dependence helps justify the parameters chosen for figure 9 .

the dispersion relation when $h_{B}^{\prime}=-1$. The growth rate and phase speed appear qualitatively similar to those shown in figure 2 . One difference is that the curve appears to be shifted towards higher wavenumber, so that the maximum-growth wavenumber is $n=6$. This is consistent with the idea that the important physical process leading to the growth of instability takes place on the downslope side of the current, since, although $r_{0}$ is the same in figures 2 and 6 , the downslope side of the current traces a longer path when $h_{B}^{\prime}=-1$. So, for a given physical wavelength, the downslope side will have a higher wavenumber $n$ when $h_{B}^{\prime}=-1$. Other differences between figures 2 and 6 are that figure 6 shows a slightly smaller maximum growth rate as well as significantly smaller phase speeds, particularly at small wavenumbers.

We show in figure 7 the dependence of the maximum growth rate and corresponding wavenumber upon the gravity current half-width $a$ and the mean radius of the gravity 
current $r_{0}$. Also shown is the dependence of the scaled wave period upon $a$ and $r_{0}$. We have found that the linear instability results are relatively insensitive to the parameter $r_{\max }$, so figure 7 results have been calculated with $r_{\max }=7.5$ to allow for a wider range of $a$ and of $r_{0}$ to be investigated. Figure 7(a) indicates that gravity currents of half-width greater than or equal to the deformation radius $L$ will have similar growth rates, but those of half-widths less than $L$ will be somewhat slower-growing instabilities. Note also for figure 7(a) that the wavenumber corresponding to the maximum growth rate is 5 for all $a$ except for $a=1.25$, the largest value tested. Thus, the fastest-growing wavenumber is relatively insensitive to the current width.

The dependence of the fastest-growing wavenumber on the mean radius of the lower-layer current is shown in figure $7(b)$. The circles show the dependence when the parameters are as for the reference case (except for $r_{\max }=7.5$ ), which is shown in figure 2. The wavenumber is strongly dependent upon $r_{0}$. Assuming the effective length of the current can be taken to be $2 \pi r_{0}$, and neglecting curvature effects so that the analysis of Swaters (1991) applies to the current, the number of waves appearing along the current for alongslope wavenumber $k$ is $n=k r_{0}$, i.e. a linear dependence on $r_{0}$. Swaters (1991) quotes the maximum-growth wavenumber for $a=1.0$ and $\mu=2.0$ to be $k=1.421$. Shown in figure $7(b)$ is the line $n=1.421 r_{0}$, as well as the dependence of the fastest-growing wavenumber on $r_{0}$ when $a=1.0$ and $\mu=2.0$. It is worth noting that the wavenumbers fall below the values one would expect if curvature effects are ignored. This implies that the effective length of the current should not be taken as $2 \pi r_{0}$, but rather some length intermediate to $2 \pi r_{0}$ and $2 \pi\left(r_{0}-a\right)$. That is, the effective radius of the current is on the downslope side of $r_{0}$. This is further evidence that it is the downslope side of the current that is important in the instability.

Figures $7(c)$ and $7(d)$ show that the scaled wave period, $1 /\left(c_{R} n\right)$ where $c_{R}$ and $n$ correspond to the most unstable mode, is relatively insensitive to the lower-layer half-width $a$ and the mean lower-layer radius $r_{0}$. This allows us to have confidence that our choice of parameter values does not strongly affect the results when we compare in figure 9 the scaled wave period to laboratory data.

In figure $7(a)$, the parameter range of $a$ which we examined was such that the necessary condition for the existence of a high-wavenumber cutoff (3.23) was always satisfied. It is interesting to note that the condition to ensure convergence of at least one of the two Frobenius series used to calculate $\eta_{1}(r)$ and $\eta_{2}(r)$ (see the discussion before (4.5)) for all possible choices of complex phase speed $c$ is precisely the same as the necessary condition for the existence of a high-wavenumber cutoff. That is, if the current width $a$ is such that $a>r_{0} / 3$, which is the regime in which we cannot prove that a high-wavenumber cutoff exists, then there exist $c$ for which neither the Frobenius series expanded about $r=r_{0}$ nor the one expanded about $r=1 / c$ converge for some $r_{0}-a \leqslant r \leqslant r_{0}+a$. We have not been able to identify any physical reason why the two conditions should coincide.

To investigate whether the high-wavenumber cutoff exists outside the parameter range for which its existence has been proven, we have performed linear stability calculations including the regime $a>r_{0} / 3$ (specifically, for $r_{0}=4.75$ and $1.0 \leqslant a \leqslant$ 3.1), with ordinary differential equation solution by the method of Frobenius replaced by numerical methods such as the fourth-order Runge-Kutta method. We have observed that the high-wavenumber cutoff follows a trend towards lower wavenumbers as $a$ increases, both for $a<r_{0} / 3$ and $a \geqslant r_{0} / 3$. This implies that the high-wavenumber cutoff does indeed exist beyond the range of parameters for which we have proven. This was confirmed by a numerical simulation of the full model (see $\S 6$ for numerical method description), which showed low-wavenumber mode growth for a wide lower 

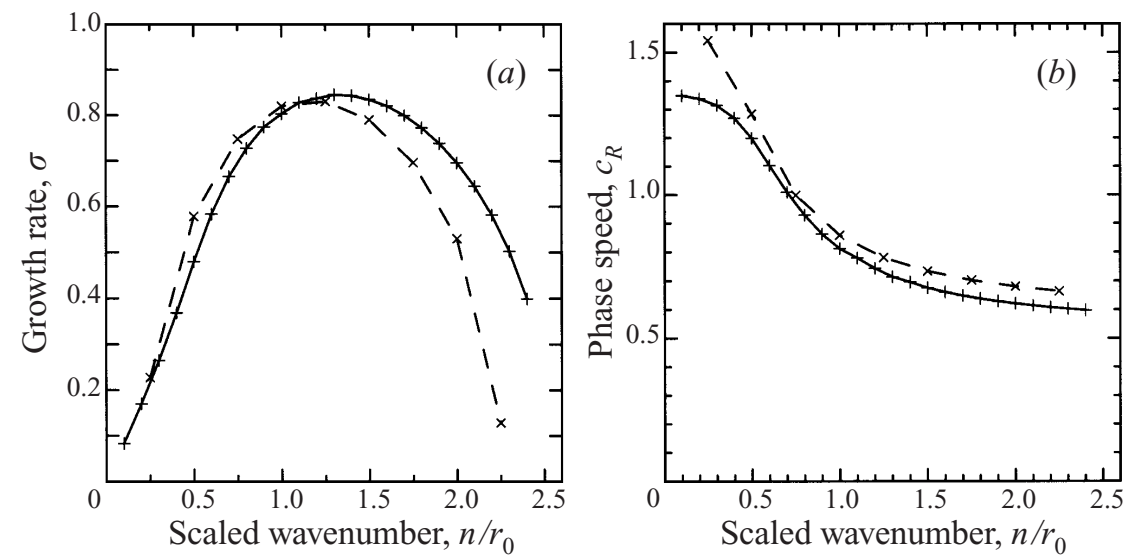

FIGURE 8. Growth rate and phase velocity versus scaled wavenumber $n / r_{0}$ for $r_{0}=4$ (dashed lines, $\times$ ) and $r_{0}=10$ (solid lines, + ). Parameters are $\mu=2, a=1$ and $r_{\max }=r_{0}+2 a$.

layer (for $r_{0}=3$ and $a=2$, the $n=2$ mode was dominant). Of course, since one can only check a finite number of parameters numerically, this does not constitute proof, but merely suggestive evidence.

The behaviour in the rectangular case should be recovered as the radius of the tank becomes large. Mathematically, this is done by introducing the transformation

$$
c \rightarrow \tilde{c} / r_{0}, \quad n \rightarrow k r_{0}, \quad \theta \rightarrow x / r_{0}, \quad r \rightarrow r_{0}-y,
$$

then taking the limit as $r_{0} \rightarrow \infty$. Then $\tilde{c}, k, x$, and $y$ correspond to the phase velocity, wavenumber, along-current coordinate, and cross-current coordinate of the rectangular case, respectively. Introducing this transformation in (4.2) and (4.3) and taking the limit as $r_{0} \rightarrow \infty$, one obtains

$$
\begin{gathered}
\eta_{y y}-\left\{k^{2}-\tilde{c}^{-1}+2 \mu\left[a^{2} \tilde{c}(\tilde{c}-1)\right]^{-1} y\right\} \eta \quad \text { for }|y|<a, \\
\eta_{y y}-\left\{k^{2}-\tilde{c}^{-1}\right\} \eta \quad \text { for }|y|>a,
\end{gathered}
$$

which are exactly the normal mode equations found by Swaters (1991).

Figure 8 shows the growth rate curve for $r_{0}=4$, and $r_{0}=10$ with the other parameter values of $\mu=2, a=1$, and $r_{\max }=r_{0}+2 a$, which were chosen as such to facilitate comparison with Swaters (1991).

It can be seen that as $r_{0}$ increases, the maximum growth rate does not change much, while the most unstable wavenumber increases slightly. This implies that the most unstable wavenumber is lower in the axisymmetric geometry than in the rectangular geometry. Thus, the instability takes place at longer wavelengths, as measured at the centre of the current. This is reasonable since the instability takes place at the downward side of the current, which is, for our bottom topography, in the direction of decreasing radius.

\section{Comparison with experimental data}

Lane-Serff \& Baines (1998), in their experimental study of dense eddies forming along a sloping bottom in a rotating system, measure $T_{i n t}$, the time interval between one eddy and the next, for a variety of parameters. They demonstrate that $T_{\text {int }} / T$, where $T$ is the period of rotation of the tank, is not at all correlated with the 
Swaters (1991) interaction parameter, $\mu$, and conclude that baroclinic instability is not an important physical process in the formation of the eddies they observed. However, Swaters (1991) does not specify what dependence $T_{\text {int }} / T$ should have on $\mu$. To facilitate comparison between model predictions and experimental observations of $T_{\text {int }}$ in the future, we document here the predictions of our model.

If the eddies are the result of baroclinic instability, then it is possible that they are formed from the nonlinear growth of the linear perturbations (Swaters 1991, 1998a). We test this hypothesis by comparing the measured time interval between eddies, $T_{\text {int }}$, to the period of the most unstable mode as determined by our linear baroclinic instability theory. We emphasize that the data of Lane-Serff \& Baines (1998) is for fully formed eddies, which are certainly not described by the linear theory of sinusoidal perturbations to a steady current. In fact, Lane-Serff \& Baines (1998) observed that the eddies formed immediately after flowing over the weir, before any steady current could be formed. Thus, the comparison we make is simultaneously a test of our model of baroclinic instability as well as the notion that the eddy time interval matches the wave period. That is, we are testing the hypothesis that baroclinic instability causes the growth of the eddies and that linear theory successfully predicts the characteristic time interval over which the eddies will tend to grow.

In dimensional units, the wavelength at a radius of $r_{0}$ is $2 \pi r_{0} L / n$, the dimensional wave speed is $r_{0} L c_{R} s f$, and the period of rotation of the tank is $T=4 \pi / f$, so that the theoretical dependence is

$$
\frac{T_{\text {int }}}{T}=\frac{1}{2 s c_{R} n},
$$

where $n$ and $c_{R}$ are the wavenumber and angular phase speed corresponding to the most unstable mode. Since the model does not explicitly contain $s$, we test (5.1) by comparing $2 s T_{i n t} / T$, as measured by Lane-Serff \& Baines (1998), to $1 /\left(c_{R} n\right)$, as predicted by the linear stability theory of the previous section (see figure $4 d$ ).

For a given value of $\mu$, we found $c_{R}$ and $n$ for the most unstable mode of a steady coupled-front current configured as shown in figure 1 and as given by (4.1), with parameter values of $a=0.75, r_{\max }=2 \pi$ and $r_{0}=r_{\max }-2 a \approx 4.783$ (corresponding to the solid dot in figure 7). These parameters were chosen such that $a$ is $O(1)$, while the front locations are well away from $r=0$ and $r=r_{\max }$, i.e. to produce typical linear stability results. With these parameters, the dimensional width of the current (and therefore the eddy diameter as well) is assumed to be on the order of the Rossby deformation radius of the upper layer. We are confident that the results are not sensitive to these parameter choices since $1 /\left(c_{R} n\right)$ is nearly constant as $a$ and $r_{0}$ vary, as shown in figures $7(c)$ and $7(d)$, respectively, and none of the stability characteristics depend strongly on $r_{\max }$.

The results are displayed in figure 9, where the laboratory measurements are represented by discrete points, and the theoretical prediction is represented by the solid line. That is, the discrete points correspond to plotting $2 s T_{\text {int }} / T$ versus $\mu$ as determined by the data of Lane-Serff \& Baines (1998), and the solid line is our theoretical prediction of $1 /\left(c_{R} n\right)$ versus $\mu$ for the most unstable mode.

The data of Lane-Serff \& Baines (1998) are for fully formed eddies, whereas the theoretical prediction is for the most unstable mode of an idealized steady coupledfront current, as calculated from linear theory. Despite this difference, the data and the theory are seen to agree quantitatively for several data points. Moreover, it is precisely those data for which the scaled slope parameter $s$ is smallest (i.e. the solid dots in figure 9) that agree with the predictions of the model. Since this model was 


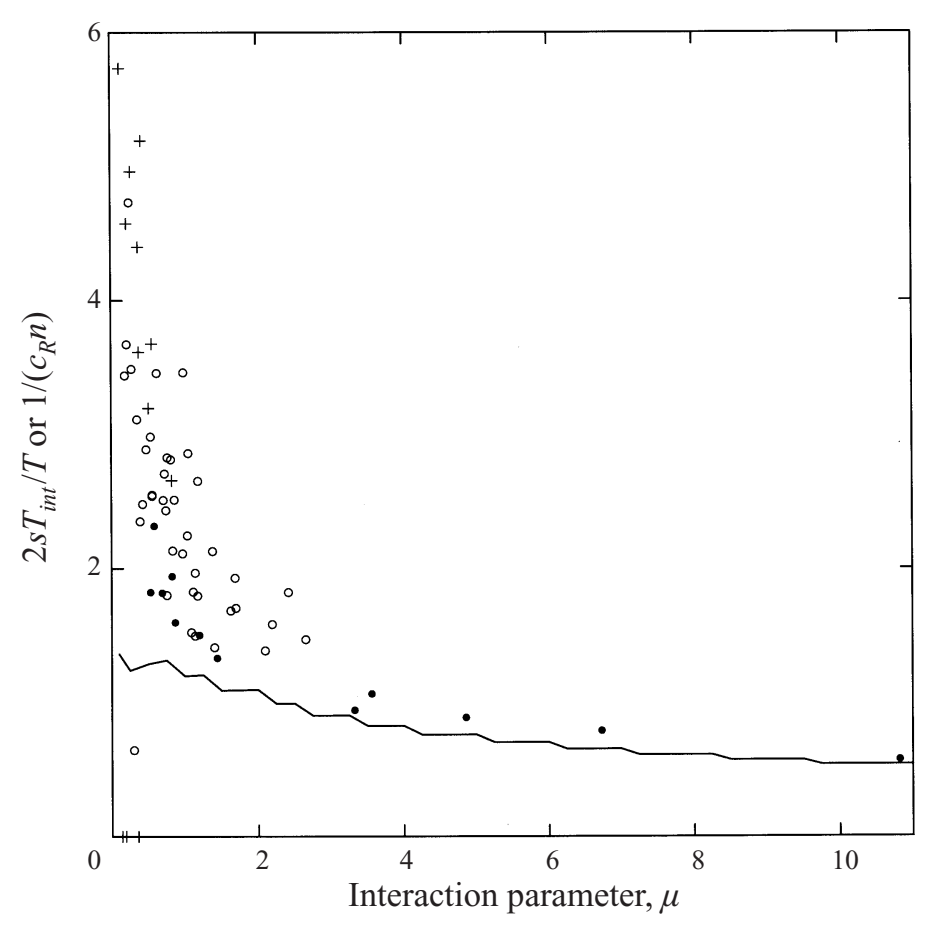

FIGURE 9. Comparison of predictions of linear theory with laboratory data of Lane-Serff \& Baines (1998). Filled circles, open circles and plus symbols correspond to laboratory data for $2 s T_{\text {int }} / T$ for which $s \leqslant 0.2,0.2<s \leqslant 0.5$ and $s>0.5$, respectively, plotted as a function of $\mu$. The solid line shows $1 /\left(c_{R} n\right)$ for the fastest growing mode at each $\mu$, as predicted by linear instability theory.

derived via an asymptotic expansion in $s$ for $s \ll 1$, it does not apply in the limit as $s \rightarrow 1$, and this is also reflected in figure 9 .

The fact that there is quantitative agreement between the linear instability analysis of this model and experimental data on travelling eddies supports the hypothesis of Swaters $(1991,1998 a)$ that baroclinic instability is indeed the physical mechanism that leads to the formation of these eddies. Lane-Serff \& Baines (1998) provide evidence that the eddies were formed through vortex stretching effects. This is consistent with our observations since the dynamics of the upper layer in this model are driven by vortex tube stretching.

Albeit indirect, figure 9 shows the first experimental evidence confirming the baroclinic instability predictions of the Swaters (1991) model. However, for the same data set, Lane-Serff \& Baines (1998) observe that the propagation speed of the eddies does not agree well with the Nof (1983) speed, which is the dependence this model predicts. Clearly, more complete experimental tests of this model are desirable. In particular, laboratory studies can answer questions such as: over what range of $s$ does this model apply?

\section{Numerical results}

The method used to integrate the model forward in time is similar to the method used by Swaters $(1998 a, b)$. The model is rewritten as

$$
q_{t}+J\left(\eta, q+h_{B}\right)=0
$$




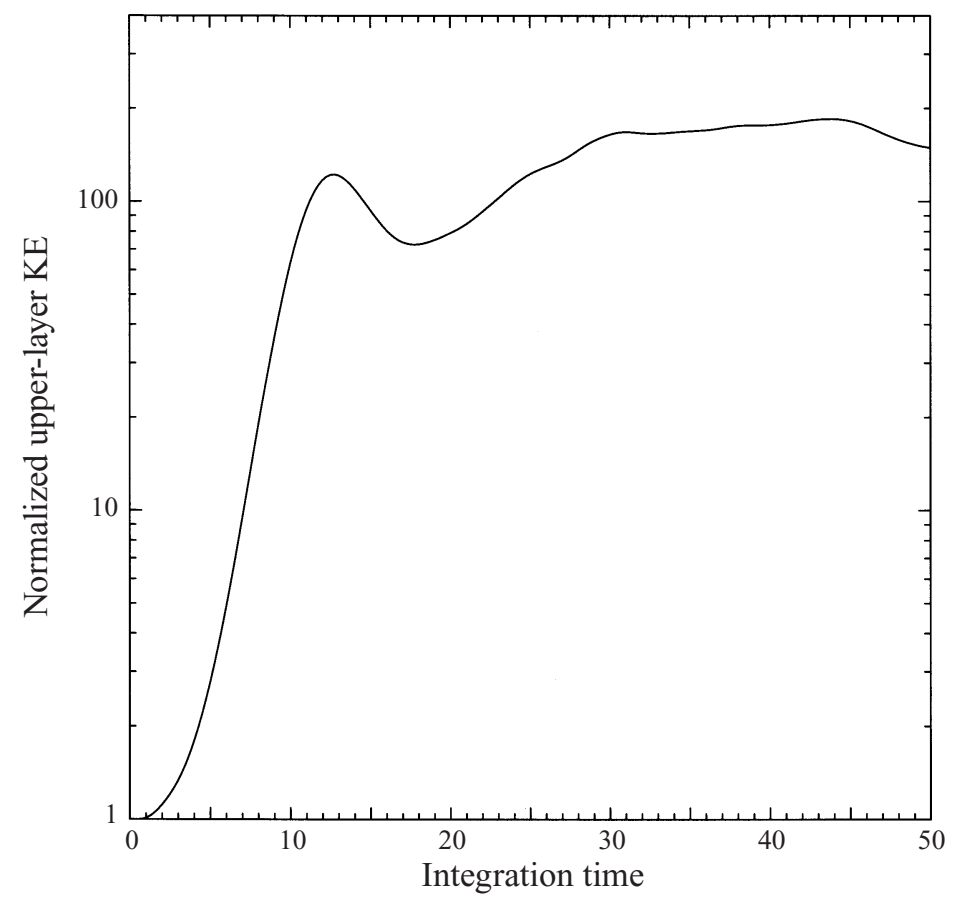

FIGURE 10. $\langle K E\rangle$ versus integration time for the numerical simulation.

$$
\begin{gathered}
h_{t}+J\left(\eta+h_{B}, h\right)=v \Delta h, \\
\Delta \eta=q-h,
\end{gathered}
$$

where $\mu$ has been set explicitly to unity, and numerical friction of the form $v \Delta h$ was added to the lower-layer equation to suppress high-wavenumber features in the solutions. The friction coefficient was taken to be $10^{-3}$ for all the simulations reported here. Equations (6.1) and (6.2) were integrated using a second-order leapfrog method with the Arakawa (1966) finite difference scheme used for the Jacobian. A Robert filter (Asselin 1972) with coefficient 0.005 was used to suppress the computational mode. Then, (6.3) yielded $\eta$ at each time step via a Poisson equation direct solver.

To avoid the $1 / r$ singularity in the polar form of the Jacobian terms, these equations were expressed in rectangular coordinates for their numerical integration. Thus, the geometry used was a $256 \times 256$ point grid in the domain

$$
\Omega=\{(x, y) \mid-2 \pi \leqslant x \leqslant 2 \pi,-2 \pi \leqslant y \leqslant 2 \pi\},
$$

with Dirichlet boundary conditions. Despite the fact that the numerical integration was performed in rectangular coordinates, the solution found converges to the true solution for cylindrical coordinates so long as the bottom topography and the initial conditions were appropriate for cylindrical coordinates, and so long as the solution found had all the relevant behaviour remaining far from the corners of the rectangular domain, which had to be checked a posteriori.

The bottom topography and the initial condition for the bottom current were slightly different than for the analysis of the linearized model, to ensure smooth derivatives everywhere. The bottom topography was hyperbolic, with the linear slope as asymptotes,

$$
h_{B}(r)=\left(r^{2}+B\right)^{1 / 2}-2 \pi \text {, }
$$


(a)
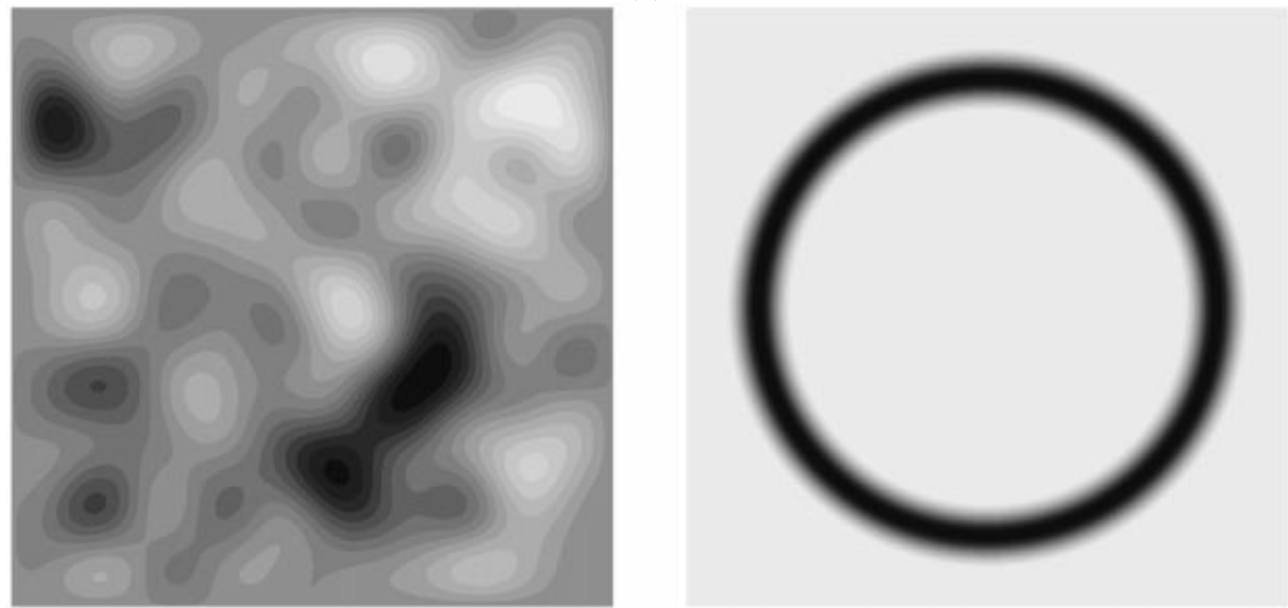

(b)
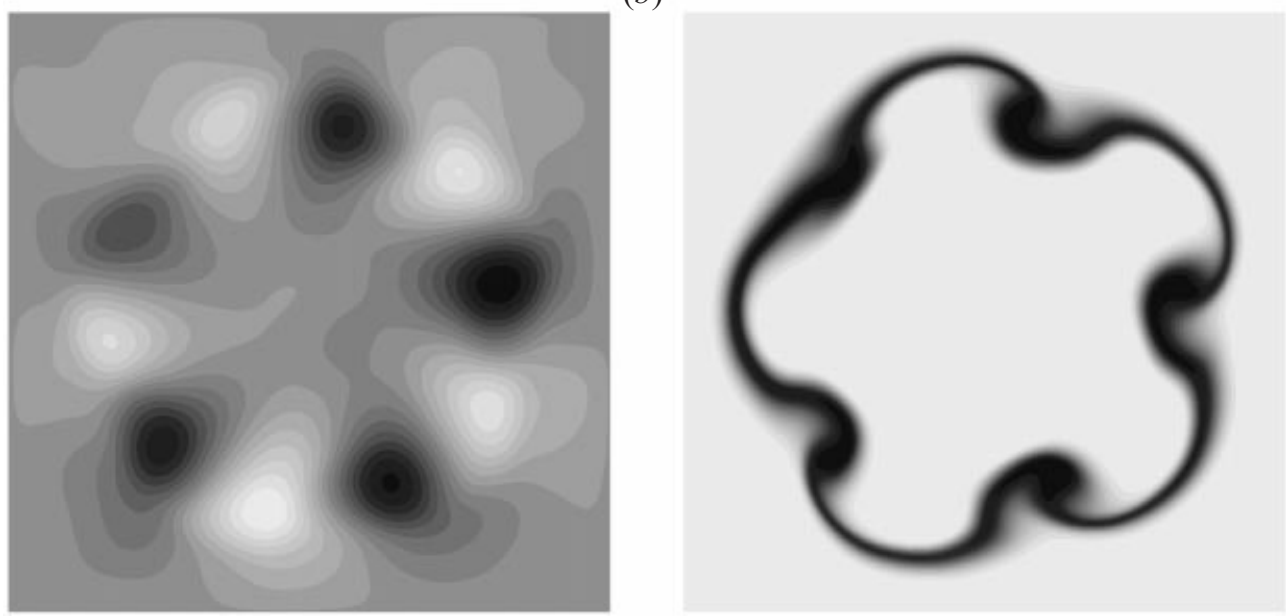

Figure 11 $(a, b)$. For caption see opposite.

where $B$ was chosen to be 0.628 . This choice of bottom topography possesses smooth derivatives of all orders, while $h_{B}^{\prime}$ remains within $3 \%$ of unity for all $3.2 \leqslant r \leqslant r_{\max }=2 \pi$. The initial condition for the bottom current was

$$
h_{0}=h(r, \theta, 0)= \begin{cases}\left\{1+\cos \left[\pi\left(r-r_{0}\right) / a\right]\right\} / 2 & \text { for }\left|r-r_{0}\right| \leqslant a \\ 0 & \text { for }\left|r-r_{0}\right|>a,\end{cases}
$$

where $a=0.75$ and $r_{0}=2 \pi-2 a$ were typical values for the simulations reported here. No initial perturbation was introduced into the lower layer. The lower-layer height at each gridpoint in the domain was allowed to evolve forward in time as determined by the model equations already stated, with no special treatment necessary to deal with the evolution of the incropping location. At gridpoints where roundoff error led to unphysical negative values of the lower layer height, the height was reset to zero.

The destabilization was initiated by the introduction of perturbations into the upper layer. The initial condition for the upper-layer stream function is a linear superposition 
(c)
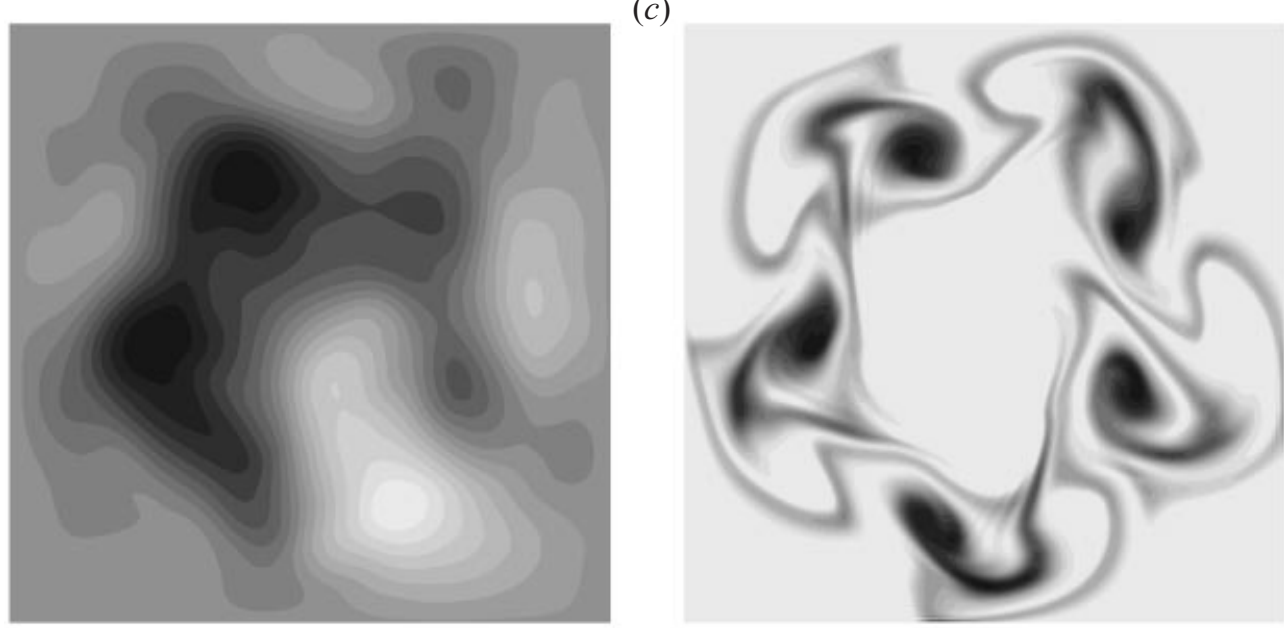

(d)
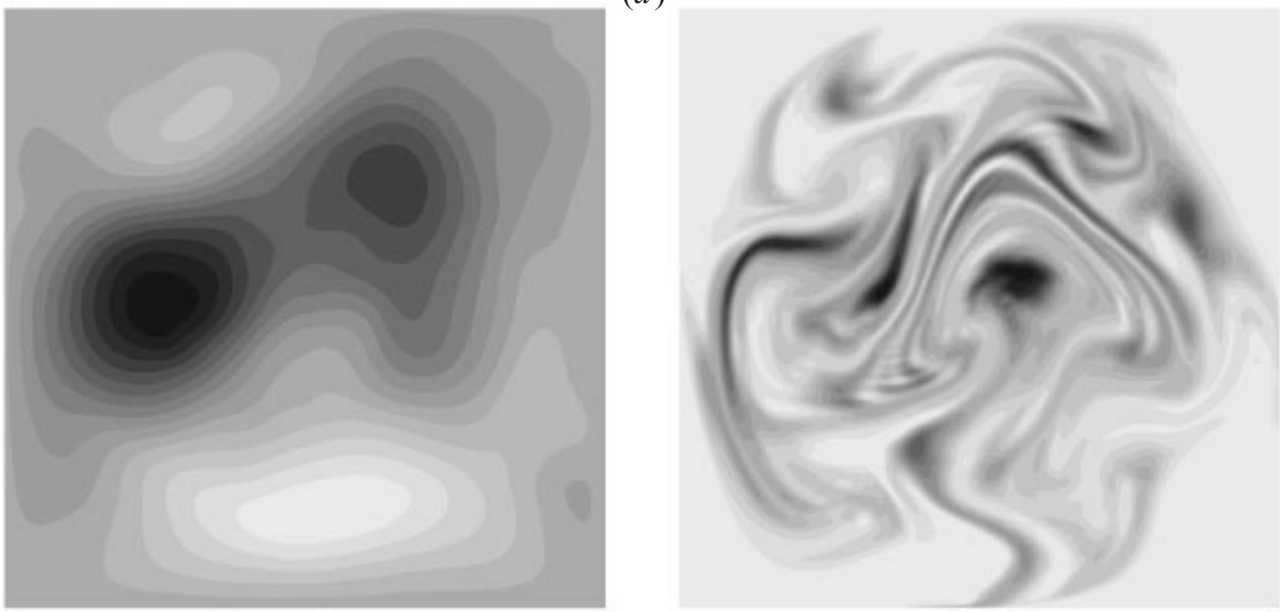

FIGURE $11(c, d)$. Upper-layer stream function (left) and lower-layer height (right) at times $t=(a)$ 0, (b) 10, (c) 20 and $(d)$ 40. Darker shades denote larger values. Lower-layer ranges are $(0.0,1.0)$, $(0.0,0.96),(0.0,0.96),(0.0,84)$, and upper-layer ranges are $(-0.07,0.07),(-0.52,0.65),(-0.74,0.9)$ and $(-1.05,1.89)$ for times $0,10,20$ and 40 , respectively.

of a range of wavelengths in the $x$ - and $y$-directions, with random amplitudes and phase shifts. For the parameters used in these simulations, wavenumbers in the range 1 to 10 were chosen because the most unstable wavenumber as determined by linear theory is $n=5$. The amplitudes for these modes making up the initial upper-layer stream function are such that the upper-layer kinetic energy is $1 \%$ the potential energy of the lower layer, i.e.

$$
\frac{\iint_{\Omega} \nabla \eta_{0} \cdot \nabla \eta_{0} \mathrm{~d} x \mathrm{~d} y}{\iint_{\Omega} h_{0}^{2} \mathrm{~d} x \mathrm{~d} y}=10^{-2} .
$$

The initial condition for $q$, the upper-layer vorticity, is calculated from $h_{0}$ and $\eta_{0}$ through (6.3). 
Figure 10 displays the evolution of the area-averaged upper-layer kinetic energy,

$$
\langle K E\rangle(t)=\frac{\iint_{\Omega} \nabla \eta \cdot \nabla \eta \mathrm{d} x \mathrm{~d} y}{\iint_{\Omega} \nabla \eta_{0} \cdot \nabla \eta_{0} \mathrm{~d} x \mathrm{~d} y} .
$$

The initially exponential increase in $\langle K E\rangle$ (which, due to the logarithmic vertical axis in figure 10, appears as a linear relationship between $t=5$ and $t=10$ ) suggests that the instability is developing in a manner that is consistent with the linear theory. However, it is seen that $\langle K E\rangle$ soon saturates, after which it does not grow further. Since the growth of $\langle K E\rangle$ is at the expense of the release of potential energy of the lower layer, the saturation of $\langle K E\rangle$ corresponds to the point at which the lower layer has completely slumped into the centre of the tank, at which point it can lose no more potential energy (see figure 11d).

Quantitatively, while linear theory predicts an exponential growth rate for $\langle K E\rangle$ of 1.02 (twice the maximum growth rate in figure 2), the measured growth rate for the curve seen in figure 10 is 0.70 . To test for the source of the discrepancy, we have performed further numerical simulations, adjusting: the lower-layer height profile, $h_{0}$, from sinusoidal to parabolic; the bottom topography, $h_{B}$, to exactly linear; the initial perturbation amplitude, as expressed through the ratio of upper-layer kinetic energy to lower-layer potential energy (see (6.6)); and which modes are excited initially, from a random superposition of wavenumbers $1 \leqslant n \leqslant 10$ to $n=5$ only. We have found that changing only $h_{0}$ and $h_{B}$ yields a lower growth rate $(0.77)$ than does changing the initial perturbation amplitude to $10^{-4}(0.81)$ or initializing only with the $n=5$ mode (0.84). Making all these changes simultaneously yields a growth rate of 0.95 . Thus, we conclude that, while the growth rate depends somewhat on the shapes of $h_{0}$ and $h_{B}$, it is the nonlinear interaction of the various modes present that most significantly contribute to the quantitative difference in the growth rates as predicted by the linear theory of $\S 4$ and the numerical simulations of this section.

Figure 11 shows snapshots of the upper-layer stream function and the lower-layer height at times $t=0,10,20$ and 40 . At $t=10$, the $n=5$ mode is clearly seen in both the upper-layer stream function and the lower-layer height, and these match qualitatively well with the linear theory predictions (see figure 3 ). Note how the lowerlayer current indeed amplifies on the downslope side more than on the upslope side. By $t=20$, the motion is well into the nonlinear regime, judging from the relatively complex form the lower layer takes. The nonlinearity is also indicated in figure 10, where it can be seen that the kinetic energy is no longer in the exponentially growing stage. Five distinct plumes of lower fluid have emerged from the $n=5$ wave. By $t=40$, they have already disappeared and the fluid seems thoroughly disorganized.

In order to follow the evolution of the azimuthal modes with time, we calculate at each time step the radially averaged azimuthal spectrum $S(n, t)$ for the upper-layer stream function. To do this, we first find the $n$th azimuthal Fourier mode at a fixed radius

$$
\tilde{\eta}(r, n, t)=(2 \pi)^{-1} \int_{0}^{2 \pi} \eta(r, \theta, t) \exp (-\mathrm{i} n \theta) \mathrm{d} \theta .
$$

We then form the modulus and integrate out the $r$-dependence

$$
S(n, t)=\int_{0}^{r_{\max }}|\tilde{\eta}(r, n, t)| r \mathrm{~d} r
$$




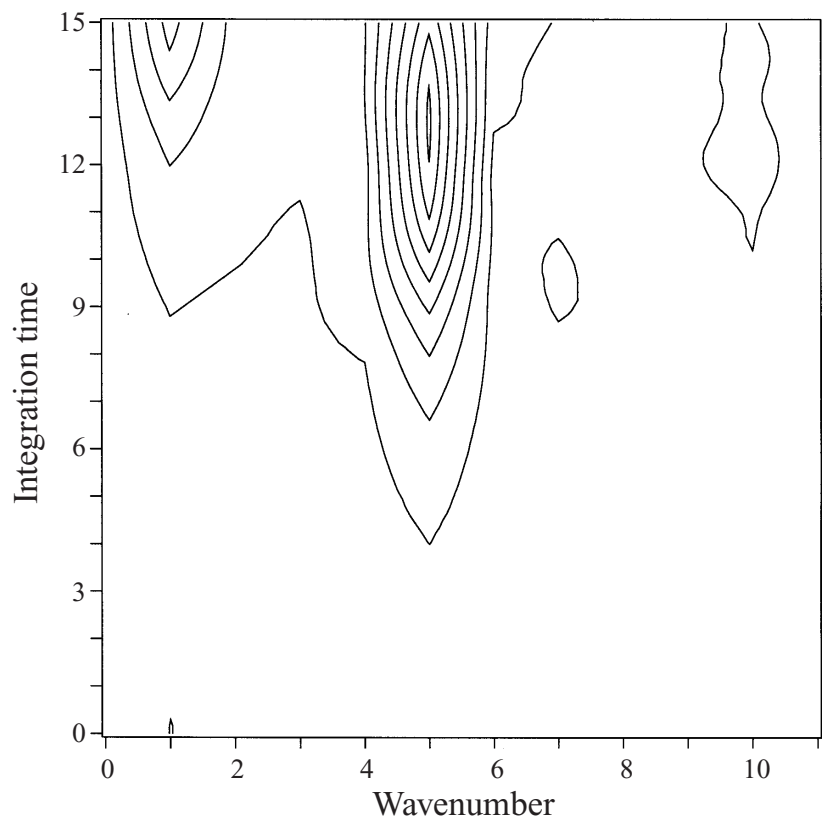

FIGURE 12. Radius-averaged azimuthal spectrum versus time.

where the extra factor of $r$ in the integrand is inserted to account for the fact that modes occurring at larger radii span more area. The azimuthal spectrum is plotted in figure 12 versus wavenumber and time.

It is readily seen that the $n=5$ mode is the dominant one. The $n=1$ mode starts growing significantly after about $t=12$. Thereafter, the $n=5$ mode starts to decay, seemingly losing its energy to the low-wavenumber modes. We have found good agreement between the numerical simulations of this model and the linear instability analysis for other parameter values as well. For example, figure 13 shows images from two simulations in which the mean radius of the lower layer $r_{0}$ is varied to determine the effect upon the fastest-growing wavenumber. The parameters were chosen to match corresponding data points shown in figure $7(b)$. The linear results predict $n=3$ for $r_{0}=3$, and this agrees very well with figure 13(a). The linear results predict $n=6$ for $r_{0}=6$, and the corresponding numerical simulation images are shown in figure $13(b)$. The agreement is not as obvious as in the lower-wavenumber cases. One can count seven plumes in the lower-layer, but they are not of uniform wavelength. There are indeed six regions of negative upper-layer stream function, with two of very small amplitude. Seven regions of positive upper-layer stream function are present, but two are very weak. The non-uniformity of the wave magnitudes is most likely due to the random initial conditions.

\section{Summary}

The baroclinic stability characteristics of axisymmetric rotating gravity currents have been determined. The model we have used is an adaptation of the model by Swaters (1991) which corresponds to a subinertial approximation to the two-layer shallow water equations which filters out barotropic instability and focuses on the 


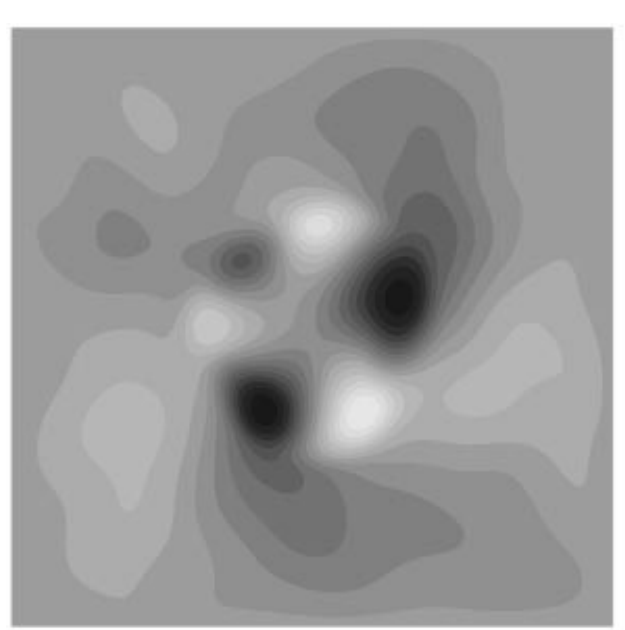

(a)
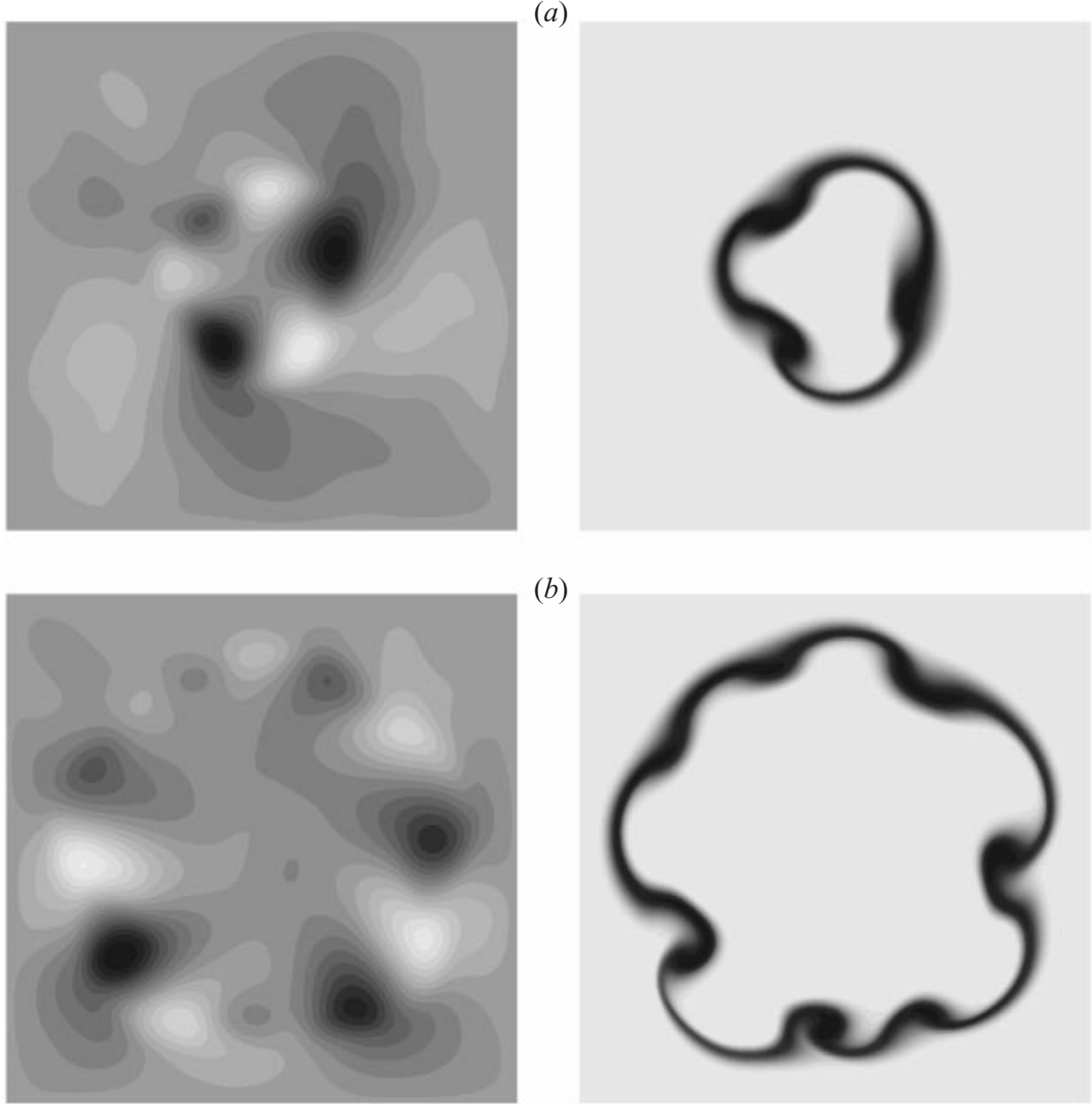

(b)

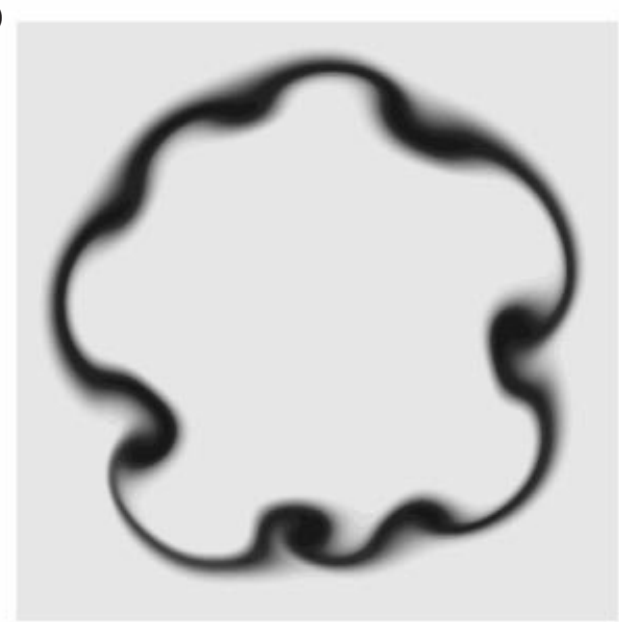

FIGURE 13. Upper-layer stream function (left) and lower-layer height (right) for runs identical to the one shown in figure 11, except that $r_{\max }=7.5$ and $r_{0}=3(a)$ and $r_{0}=6(b) . r_{0}=3$ run shown at $t=12$, and $r_{0}=6$ run shown at $t=10$. Darker shades denote larger values. Lower-layer ranges are $(0.0,0.94)$ and $(0.0,0.97)$, and upper-layer ranges are $(-0.34,0.48)$ and $(-0.57,0.79)$, for $r_{0}=3$ and $r_{0}=6$, respectively.

baroclinic dynamics while allowing for finite-amplitude thickness variations in the gravity current.

Several general theoretical results were obtained including necessary conditions for instability, a semicircle-like theorem, bounds on the growth rate and phase velocity and a high-wavenumber cutoff. The instability proceeds by the perturbations extracting the available gravitational potential energy associated with the cross-slope position of the gravity current. Equivalently, this may thought of as the net up-topographicgradient transport of heat, i.e. classical baroclinic instability. From the modal point of view, the instability corresponds to the coalescence of two azimuthally propagating topographic Rossby waves in the overlying fluid.

The interaction parameter, which is the ratio of the scale height of the bottom current to the scale height of the bottom topography, strongly influenced the instabil- 
ity. Dynamically, this parameter measures the strength of the destabilizing effects of baroclinic stretching to the stabilizing effect of the background topographic vorticity gradient. For sufficiently large, but nevertheless not unphysical, values of the interaction parameter, a dipole-shaped instability was found which resembles that described by Griffiths et al. (1982).

The predictions of the model were compared with laboratory data. Despite the fact that the theoretical predictions were for linear waves whereas the data were for travelling eddies, good agreement was seen, especially in the small-s regime for which this model was derived. This suggests that the baroclinic instability of the waves may be an important physical process in the generation of such eddies, even when no steady current was present beforehand.

The fully nonlinear equations of the model were integrated numerically. The behaviour of the system at early times was seen to be qualitatively consistent with the linear results. The instabilities were preferentially amplified in the downslope direction, which pointed to the fact that the instability was baroclinic in nature, driven by the release of potential energy of the bottom current. The perturbations to the downslope incropping develop into downslope-propagating plumes which subsequently reach the deepest part of the tank, at which point no more potential energy can be released.

It remains for future work to thoroughly compare the predictions of this model with experimental observations. Laboratory investigations of dense flows on a sloping bottom tend to be localized sources on the slope (producing a train of eddies immediately or not at all) or axisymmetric outflow from a central source. An experimental study which has not, to our knowledge, been performed yet is the one where the current is an initially steady coupled-front dense current on a sloping bottom. Since experiments taking into account Coriolis effects are typically performed in a cylindrical rotating tank, it is hoped that the study of this model explicitly in such a geometry will facilitate the conduction and subsequent interpretation of experiments on coupled-front geostrophic flows with bottom topography.

Preparation of this paper was supported in part by Natural Sciences and Engineering Research Council (NSERC) Research Grants awarded to G. E. S. and by a NSERC Postgraduate Scholarship and a Killam Doctoral Scholarship awarded to P.F. C.

\section{REFERENCES}

ArakaWA, A. 1966 Computational design for long-term numerical integration of the equations of fluid motion: Two-dimensional incompressible flow. J. Comput. Phys. 1, 119-143.

Asselin, R. A. 1972 Frequency filter for time integrations. Mon. Weather Rev. 100, 487-490.

Chapman, D. C. \& Gawarkiewicz, G. 1995 Offshore transport of dense shelf water in the presence of a submarine canyon. J. Geophys. Res. 100 (C7), 13373-13387.

Condie, S. A. 1995 Descent of dense water masses along continental slopes. J. Mar. Res. 53, 897-928.

EtLing, D. \& Chabert D'Hieres, G. 1997 Laboratory experiments on density currents on a slope in a rotating system. In Proc. 11th AMS Conf. on Atmospheric and Oceanic Fluid Dyn., pp. 211-212.

Gawarkiewicz, G. \& Chapman, D. C. 1995 A numerical study of dense water formation and transport on a shallow, sloping continental shelf. J. Geophys. Res. 100 (C3), 4489-4507.

Griffiths, R. W. 1986 Gravity currents in rotating systems. Ann. Rev. Fluid Mech. 18, 59-89.

Griffiths, R. W., Killworth, P. D. \& Stern, M. E. 1982 Ageostrophic instability of ocean currents. J. Fluid Mech. 117, 343-377.

Houghton, R. W., Schlitz, R., Beardsley, R. C., Butman, B. \& Chamberlin, J. C. 1982 The 
middle Atlantic bight pool: evolution of the temperature structure during 1979. J. Phys. Oceanogr. 12, 1019-1029.

JiANG, L. \& GARWOOD JR., R. W. 1995 A numerical study of three-dimensional dense bottom plumes on a Southern Ocean continental slope. J. Geophys. Res. 100 (C9), 18471-18488.

JiANG, L. \& GARwoOD JR., R. W. 1996 Three-dimensional simulations of outflows on continental slopes. J. Phys. Oceanogr. 26, 1214-1233.

Karsten, R. H. \& Swaters, G. E. 1996 Nonlinear stability of baroclinic fronts in a channel with variable topography. Stud. Appl. Maths 96, 183-199.

Karsten, R. H., Swaters, G. E. \& Thomson, R. E. 1995 Stability characteristics of deep-water replacement in the Strait of Georgia. J. Phys. Oceanogr. 25, 2391-2403.

Lane-SerfF, G. F. \& Baines, P. G. 1998 Eddy formation by dense flows on slopes in a rotating fluid. J. Fluid Mech. 363, 229-252.

LeBlond, P. H., Ma, H., Doherty, F. \& Pond, S. 1991 Deep and intermediate water replacement in the Strait of Georgia. Atmos.-Ocean 29, 288-312.

Maxworthy, T. 1997 Convection into domains with open boundaries. Ann. Rev. Fluid Mech. 29, $327-371$.

Mory, M., Stern, M. E. \& Griffiths, R. W. 1987 Coherent baroclinic eddies on a sloping bottom. J. Fluid Mech. 183, 45-62.

Nagata, Y., Kimura, R., Honji, H., Yamazaki, Y., Kawaguchi, K. \& Hosoyamada, T. 1993 Laboratory experiments of dense water descending on continental slope. In Deep Ocean Circulation, Physical and Chemical Aspects (ed. T. Teramoto), pp. 333-350. Elsevier.

NoF, D. 1983 The translation of isolated cold eddies on a sloping bottom. Deep-Sea Res. 30, 171-182.

PedLosky, J. 1987 Geophysical Fluid Dynamics, 2nd Edn. Springer.

Poulin, F. J. \& Swaters, G. E. 1999a Sub-inertial dynamics of density-driven flows in a continuously stratified fluid on a sloping bottom. I. Model derivation and stability characteristics. Proc. R. Soc. Lond. A 455, 2281-2304.

Poulin, F. J. \& Swaters, G. E. 1999b Sub-inertial dynamics of density-driven flows in a continuously stratified fluid on a sloping bottom. II. Isolated eddies and radiating cold domes. Proc. R. Soc. Lond. A 455, 2305-2329.

Poulin, F. J. \& Swaters, G. E. 1999c Sub-inertial dynamics of density-driven flows in a continuously stratified fluid on a sloping bottom. Part 3. Nonlinear stability theory. Can. Appl. Maths Q. 7, 49-68.

Smiтh, P. C. 1977 Experiments with viscous source flows in rotating systems. Dyn. Atmos. Oceans 1, $241-272$

Spall, M. A. \& Price, J. F. 1998 Mesoscale variability in Denmark Strait: the PV outflow hypothesis. J. Phys. Oceanogr. 28, 1598-1623.

Swaters, G. E. 1991 On the baroclinic instability of cold-core coupled density fronts on a sloping continental shelf. J. Fluid Mech. 224, 361-382.

Swaters, G. E. 1993 Nonlinear stability of intermediate baroclinic flow on a sloping bottom. Proc. R. Soc. Lond. A 442, 249-272.

Swaters, G. E. $1998 a$ Numerical simulations of the baroclinic dynamics of density-driven coupled fronts and eddies on a sloping bottom. J. Geophys. Res. 103, 2945-2961.

Swaters, G. E. $1998 b$ Dynamics of radiating cold domes on a sloping bottom. J. Fluid Mech. 364, $221-251$.

Swaters, G. E. \& Flierl, G. R. 1991 Dynamics of ventilated coherent cold eddies on a sloping bottom. J. Fluid Mech. 223, 565-587.

Ungarish, M. \& Huppert, H. E. 1998 The effects of rotation on axisymmetric gravity currents. $J$. Fluid Mech. 362, 17-51.

Waugh, D. W. \& Dritschel, D. G. 1991 The stability of filamentary vorticity in two-dimensional geophysical vortex-dynamics models. J. Fluid Mech. 231, 575-598.

Whitehead, J. A., Stern, M. E., Flierl, G. R. \& Klinger, B. A. 1990 Experimental observations of baroclinic eddies on a sloping bottom. J. Geophys. Res. 95 (C6), 9585-9610.

Whitehead, J. A. \& Worthington, L. U. 1982 The flux and mixing rates of Antarctic Bottom Water within the North Atlantic. J. Geophys. Res. 87, 7903-7924.

Zatsepin, A. G., Didkovski, V. L. \& Semenov, A. V. 1998 Self-oscillatory mechanism of inducing a vortex structure by a stationary local source over a sloping bottom in a rotating fluid. Oceanology (Engng Trans.) 38, 43-50. 
Zatsepin, A. G., Kostyanoi, A. G. \& Semenov, A. V. 1996 An axisymmetric density current over a sloping bottom in a rotating fluid. Oceanology (Engng Trans.) 36, 311-316.

Zoccolotti, L. \& Salusti, E. 1987 Observations on a very dense marine water in the Southern Adriatic Sea. Continent. Shelf Res. 7, 535-551. 